\title{
Evaluation of Stochastic Particle Dispersion Modeling in Turbulent Round Jets
}

\author{
Guangyuan Sun ${ }^{\mathrm{a}}$, John C. Hewson ${ }^{\mathrm{b}}$, David O. Lignell ${ }^{\mathrm{a}, 1, *}$ \\ a350 CB, Brigham Young University, Provo, UT 84602, USA \\ ${ }^{b}$ Fire Science and Technology Department, Sandia National Laboratories, Albuquerque, NM, USA
}

\begin{abstract}
ODT (one-dimensional turbulence) simulations of particle-carrier gas interactions are performed in the jet flow configuration. Particles with different diameters are injected onto the centerline of a turbulent air jet. The particles are passive and do not impact the fluid phase. Their radial dispersion and axial velocities are obtained as functions of axial position. The time and length scales of the jet are varied through control of the jet exit velocity and nozzle diameter. Dispersion data at long times of flight for the nozzle diameter $(7 \mathrm{~mm})$, particle diameters (60 and $90 \mu \mathrm{m})$, and Reynolds numbers (10000 to 30000) are analyzed to obtain the Lagrangian particle dispersivity. Flow statistics of the ODT particle model are compared to experimental measurements. It is shown that the particle tracking method is capable of yielding Lagrangian prediction of the dispersive transport of particles in a round jet. In this paper, three particle-eddy interaction models (Type-I, -C, and -IC) are presented to examine the details of particle dispersion and particle-eddy interaction in jet flow.
\end{abstract}

Keywords: jet, particle dispersion, one dimensional turbulence, ODT

\section{Introduction}


required that do not resolve all flow scales in three dimensions. Many gas-particle flows have been studied in which the subgrid-scale turbulence is modeled using large eddy simulation (LES) [39, 1]. LES provides good means to capture unsteady physical features in the turbulence. The accuracy and the reliability of LES predictions depend on several factors, such as the accurate modeling of the subgrid-scale phase interactions.

A promising alternative approach is the one-dimensional turbulence (ODT) model, which is able to resolve a full range of length scales on a one-dimensional domain that is evolved at the finest time scales [16, 18]. ODT has been applied to many different homogeneous and shear-dominating reacting $[8,12,13,26,25,21]$ and nonreacting $[16,18,2,34]$ flows including homogeneous turbulence, channel flow, jets, mixing layers, buoyant plumes, and wall fires.

Schmidt et al. [31] extended the ODT model to the prediction of particle-velocity statistics in turbulent channel flow. Punati [25], and Goshayeshi and Sutherland [10, 9] studied coal combustion and particle laden jets using ODT (using a version of the Type-C model noted below). In our previous study, one version of the ODT multiphase interaction model using an instantaneous (referred to as Type-I) particle-eddy interaction (PEI) model was presented to investigate particle transport and crossing-trajectory effects in homogeneous turbulence [34]. Here, we extend this previous ODT study to shear flows and present two new PEI models to analyze the behavior of individual particles in jets at high Reynolds numbers $(R e)$. One of the models applies continuous PEI (referred to as Type-C) and the other combines instantaneous and continuous interaction features (referred to as Type-IC).

The remainder of this paper is organized as follow: first, a summary description of ODT is presented, with details of of the PEI models given. This is followed by a presentation and discussion of the results of the Type-I, -C and -IC models, including comparisons to experimental results. Sensitivity of results to the single particle model parameter is discussed, and summary and concluding remarks are given.

\section{Numerical description}

\subsection{ODT model}

One-dimensional turbulence (ODT) is a numerical method to generate realizations of turbulent flows using a stochastic model of the turbulent cascade on a one-dimensional domain [16]. The one-dimensional domain is formulated in the direction of primary velocity gradients and on which the governing equations for, e.g., mass, momentum, energy, and species conservation are solved. Most ODT applications, including that presented here, use Cartesian coordinates in which the $y, x$ and $z$ coordinates are the ODT domain-aligned, streamwise (direction for flow evolution), and spanwise directions, respectively.

The ODT model consists of two main mechanisms: diffusive advancement, and advective eddy events. The diffusive evolution on the 1D domain is governed by transport equations (described below) that omit the nonlinear advective terms, which are modeled by the eddy events. These diffusive equations dissipate velocity fluctuations and kinetic energy, though this process is only significant at diffusive scales, and the eddy events 
model the cascade of fluctuations to the dissipative scales. In general flows, nonlinear advection describes a vortex-stretching process that acts in three dimensions to transfer fluctuations to higher wave numbers and is costly to predict. In order to describe these nonlinear advective terms, ODT introduces the concept of the socalled "triplet map" that transfers fluctuations to higher wave numbers during eddy events. The triplet maps that make up the eddy events in ODT occur instantaneously. The rate of occurrence of this transfer by ODT eddy events is determined through a stochastic sampling of the evolving velocity field through a measure of the shear energy that is a function of the location on the domain and the eddy length scale (wavenumber). There are two approaches to evolve the ODT domain: (i) temporal evolution where each ODT realization is parameterized by $(y, t)$ and represents a (possibly Lagrangian) time history, and (ii) spatial evolution, where each ODT realization is parameterized by $(y, x)$. Even in predicting spatially developing flows like the jet in this case, most ODT simulations have been conducted using temporal evolution assuming a Lagrangian evolution of the flow domain to map results to the spatial evolution [12].

\subsubsection{Diffusive advancement}

In the Lagrangian frame of reference, choosing $(y, t)$ as independent variables, the governing equations are derived from the Reynolds transport theorem and advanced in time along the ODT line [21]. Since there is no mass source term, no non-convection mass flux, and uniform properties inside the grid control volumes in one dimension, the finite-volume equation applied on the grid cells for the continuity equation is

$$
\rho \triangle y=\text { constant }
$$

where the density $\rho$ is constant for the nonreacting flow considered here. The diffusive advancement evolves scalar equations of momentum (per mass) component $U_{i}$ using a conservative finite volume method written here for a given cell:

$$
\frac{d U_{i}}{d t}=-\frac{1}{\rho \triangle y}\left(\sigma_{i, e}-\sigma_{i, w}\right),
$$

where $\sigma_{i, j}$ is the viscous stress. The subscripts $e$ and $w$ represent east and west faces of the control volume. The viscous stresses for the three velocity components are represented as

$$
\sigma_{i}=-\mu \frac{d U_{i}}{d y}
$$

where $\mu$ is viscosity. The spatial derivative appearing in this equation is evaluated at cell faces using a finite difference approximation between the two neighboring cells.

\subsubsection{Eddy events}

Turbulence is characterized by a three-dimensional vortex stretching process that is modeled in ODT through a representative sequence of eddy events as introduced at the beginning of this section. This model has two key components, the triplet-map representation of the length-scale cascade and the model for the rate of triplet maps. Turbulent eddies are sampled randomly on the domain as a function of the eddy 
location, represented by their left bound, $y_{0}$, and by their size, $l$, with the triplet map occurring over the region $\left[y_{0}, y_{0}+l\right]$ for the given sample. The triplet map spatially compresses the fluid property profiles within $\left[y_{0}, y_{0}+l\right]$ by a factor of three. The original profiles are replaced with three copies of the compressed profiles, with the middle copy spatially inverted. This mapping is described by

$$
f(y)=y_{0}+ \begin{cases}3\left(y-y_{0}\right) & \text { if } y_{0} \leq y \leq y_{0}+1 / 3 l \\ 2 l-3\left(y-y_{0}\right) & \text { if } y_{0}+1 / 3 l \leq y \leq y_{0}+2 / 3 l \\ 3\left(y-y_{0}\right)-2 l & \text { if } y_{0}+2 / 3 l \leq y \leq y_{0}+l, \\ y-y_{0} & \text { otherwise. }\end{cases}
$$

where $f(y)$ and $y$ are the original fluid location and the post-triplet-map location, respectively. The fluid outside $\left[y_{0}, y_{0}+l\right]$ is unaffected. The triplet map is measure preserving and all integral properties (e.g., mass, momentum, and energy) or moments thereof are constant during a triplet map. Specifically, the kinetic energy is conserved, which is a desirable property because eddy events physically model the inviscid advection process. Immediately after the triplet map, kernel transformations are introduced that redistribute energy among the velocity components [37]. The transformations are meant to model the velocity randomization and so-called return to isotropy effect in turbulent flows. The kernel can be considered as a wave function that adds or subtracts energy from the eddy based on the amplitude of the wave. An eddy event maps the velocity component $i$ as follows:

$$
U_{i}(y) \longrightarrow U_{i}(f(y))+c_{i} K(y)
$$

where the kernel $K(y) \equiv y-f(y)$ is the displacement induced by the triplet map and integrates to zero over the eddy interval. $c_{i}$ is the kernel coefficient of $K(y)$ and is specified to ensure conservation of energy among momentum components. This form is written for constant density flows, as studied here. A variable density formulation is also available [2].

The procedure to sample and accept an eddy follows that described in [21], and a summary description is provided here. The eddy rate density for an eddy occurrence at location $y_{0}$ and length $l$ is denoted as $\lambda_{e}\left(y_{0}, l, t\right)$ and is dimensionally $\tau_{e}^{-1} l^{-2}$ where $\tau_{e}$ is an eddy time scale given in Eq. 10. The rate of all eddies at a given time is $\Lambda(t)=\iint \lambda_{e}\left(y_{0}, l, t\right) d y_{0} d l$, and the eddy PDF is defined as $P\left(y_{0}, l, t\right)=\lambda\left(y_{0}, l, t\right) / \Lambda(t)$. (In the following, the $y_{0}, l$, and $t$ functional dependecies will be presumed.) Ideally, eddies would be sampled from this PDF, with occurrence times sampled with Poisson statistics with mean rate $\Lambda$. However, this is inconvenient and computationally expensive since the two dimensional eddy distribution would have to be constructed at each timestep, with a correspondingly complex sampling procedure involving numerical inversion. Instead, we use a thinning method [19] coupled with the rejection method [24]. In a thinning process, we can sample in time as a Poisson process with mean rate $\alpha \Lambda$ where $\alpha>1$, and then accept eddies with probability $P_{a}=\Lambda / \alpha \Lambda$. In the rejection method, rather than sample from the unknown $P$, we sample 
eddies from a presumed distribution $\tilde{P}$, and accept with probability $P_{a}=P / \beta \tilde{P}$, where $\beta$ is some constant (or in general, some function) so that $P_{a}<1$ (i.e., $\beta>1$ ). Together, these give

$$
P_{a}=\frac{\Lambda}{\alpha \Lambda} \frac{P}{\beta \tilde{P}} .
$$

Now, take $\Delta t_{s}=1 / \alpha \Lambda$, insert $\Lambda P=\lambda=1 / \tau_{e} l^{2}$, and absorb $1 / \beta$ into $\Delta t_{s}$ so that $\Delta t_{s} / \beta \Rightarrow \Delta t_{s}$ (since $\alpha>1$ and $\beta>1$ are arbitrary), to give

$$
P_{a}=\frac{\Delta t_{s}}{\tau_{e} l^{2} \tilde{P}} .
$$

Note that $1 / \tau_{e} l^{2}$ in Eq. 7 gives the actual eddy rate determined from the sampled instantaneous velocity field as given below in Eq. 10. The choice of $\tilde{P}$ may affect the efficiency, but not the accuracy. We use

$$
P_{0}\left(y_{0}, l\right)=g\left(y_{0}\right) f(l) .
$$

The eddy location distribution, $g\left(y_{0}\right)$, is taken to be uniform over the domain while the eddy size distribution, $f(l)$, is assumed to be $[21]$

$$
f(l)=A_{l} \exp (-2 \tilde{l} / l),
$$

where $\tilde{l}$ is the most probable eddy size (typically 0.015 times the domain length) and $A_{l}$ is the PDF normalization constant. Eddy occurrence times are sampled as a Poisson process with mean rate $\Delta t_{s}$, with the eddy size and location sampled from $f(l)$ and $g\left(y_{0}\right)$. Each candidate eddy is accepted with probability $P_{a}$ given above. $\Delta t_{s}$ is adjusted during the simulation to ensure that the average $P_{a}$ is of order 0.02 .

The eddy time scale $\tau_{e}$ is obtained using a measure of the available energy at wavelength $l$. In the present constant density work (without buoyant or other forms of energy), $\tau_{e}$ is computed using scaling arguments to relate to the available kinetic energy, which is given by $E_{k i n}=\rho\left(U_{K}^{2}+V_{K}^{2}+W_{K}^{2}\right)[16]$,

$$
\frac{1}{\tau_{e}}=C \sqrt{\frac{2}{\rho l^{2}}\left(E_{k i n}-Z E_{v p}\right)} .
$$

To obtain $E_{k i n}$ the velocities are integrated across the kernel function $K(y)$ as

$$
U_{K}=\frac{1}{l^{2}} \int_{y_{0}}^{y_{0}+1} U(f(y)) K(y) d y .
$$

In Eq. $10, E_{v p}$ is included as a viscous penalty to restrict unphysically small eddies,

$$
E_{v p}=\rho \nu / l,
$$

where $\nu$ is the kinematic viscosity of the fluid.

Beyond the basic elements of Eq. 11 as a measure of velocity fluctuations, the form of Eq. 11 is not fixed, and other forms have been used [16]. In Eq. 10, $C$ is a constant model parameter relating the kinetic energy formed from Eq. 11 to the eddy time scale in Eq. 10. $C$ directly scales the probability of an eddy occuring as per Eq. 7. Similarly, a constant, $Z$, is introduced for the viscous energy dissipation, $E_{v p}$. $C$ 
plays an important role in the rate for the turbulent cascade and the flow evolution is sensitive to it, as the rate of evolution of the flow is directly proportional to $C . Z$ is provided more as a numerical expedient to reduce the occurrence of sub-Kolmogorov scale eddies; these small eddies affect transport less than the viscous evolution. A maximum value of $Z$ will exist above which there will be an unphysical buildup of fluctuations above the Kolmogorov scale that is visible in spectra (not shown here).

In unbounded systems, like jets, eddy events may result in the occurrence of unphysically large eddies that adversely affect the overall mixing, and a mechanism for suppressing such eddies is required. This is not normally needed for bounded systems (such as channel flows), or other cases (such as stratification) that otherwise limit the mixing. There are several mechanisms of large eddy suppression that have been developed $[12,13,18,2]$. The method favored for jet flows is an elapsed time method in which the eddy time scale $\tau_{e}$ can be compared with the simulation elapsed time $t$; eddy events are allowed only when $t \geq \beta_{l e s} \tau_{e}$, where $\beta_{\text {les }}$ is a model parameter. $\beta_{\text {les }}$ has a similar (but inverse) effect of $C[12]$ : larger values of $\beta_{\text {les }}$ suppress larger eddies, and delay the flow evolution.

In summary, there are three ODT parameters $C, Z$, and $\beta_{\text {les }}$ that control the evolution of the jet. $C$ is the eddy rate parameter and scales the time evolution of the jet. $Z$ suppresses unphysically small eddies and the overall flow is insensitive to this parameter. $\beta_{\text {les }}$ suppresses large eddies and has a similar, but inverse, effect as $C$. In the next section, we discuss the Lagrangian particle model, where an additional parameter $\beta_{p}$ is introduced.

\subsection{Lagrangian particle model}

The velocity and trajectory of particles are described by a Lagrangian approach in this study. Like the ODT treatment of the continuous fluid phase, the action of turbulent eddies is handled in a special manner, referred to here as the particle-eddy interaction (PEI), as compared with diffusive processes characterized by the standard approaches described in Sec. 2.2.1. The triplet map is implemented as an instantaneous process, and the action of the triplet map on the particle can be treated either as an instantaneous or continuous process as observed in the flow evolution coordinate. The motion of the particles is traced as they interact with a random succession of turbulent eddy motions, each of which represents a Type-I (referred to as instantaneous), Type-C (referred to as continuous), or Type-IC (referred to as instantaneous and continuous) interaction between a particle and a triplet map. In the Type-I model, the PEI is represented as an instantaneous change of the particle position and velocity in the same manner that the triplet-map itself is an instantaneous event. In the Type-C model, the PEI occurs during the flow evolution by mapping the equivalent triplet-map space-time influence to the flow evolution. In the Type-IC model, the particles undergo the Type-I PEI when they are in the eddy region at the time of the eddy occurrence, and experience the Type-C PEI if they are initially outside the eddy, but move into the eddy region during the flow evolution. Dispersive transport property statistics of particles are obtained by computing a statistically significant 
ensemble of flow realizations and particle trajectories. Schmidt [28] proposed several particle models that are similar in nature to the ones here, and implemented the Type-I model to study particle behavior in a different context [31, 30]. A version of the Type-C model was used by Punati [25] and by Goshayeshi and Sutherland $[10,9]$. In this section, we summarize the implementation of the models, and more importantly, discuss and compare different types of particle-eddy interactions.

A particle-eddy interaction occurs when both the particle and the triplet map occupy the same spacetime. To predict the interaction, a finite temporal interval and spatially cubic region, consistent with turbulence isotropy, is assumed for each eddy based on the eddy time and length scale, $\tau_{e}$ and $l$. This spatial-temporal region is referred to as the eddy box. Within the eddy box, the particle evolves in the $x, y$, and $z$ dimensions as described in the following subsections, and the PEI ends when the particle leaves the idealized eddy box or when the eddy lifetime has passed. The eddy lifetime,

$$
t_{e}=\beta_{p} \tau_{e}(y, l ; t),
$$

is related to the eddy time scale, $\tau_{e}(y, l ; t)$, but these quantities should not be expected to be equal; the proportionality between these times is represented by the parameter $\beta_{p}$.

In many flows, the particles typically leave the box at the end of the eddy lifetime, $t_{e}$, but if there is significant relative motion between particles and eddies, the particles will depart spatially. This latter spatial crossing of the eddy boundary is referred to in the literature as the crossing-trajectory effect [7]. This use of an eddy length and lifetime to predict the eddy influence on the particles is common to the stochastic approaches. In the ODT model, the fluid evolution results in a full spectrum of dynamic and flow-dependent eddy scales, as opposed to only predicting integral scale eddies (or scales sampled from some static eddy distribution). The selection of the eddy lifetime in the ODT formulation is equivalent to the selection in other modeling approaches of $t_{e}$, and a proportionality appears there between the integral turbulent time scale evaluated from, for example, the turbulent kinetic energy and its dissipation rate. In approaches we will refer to as discontinuous random walk, an eddy-velocity fluctuation is selected to act for an eddy lifetime $[40,11,33]$. Another class of models referred to as continuous random walk approaches sample fluctuating velocity increments $[4,41,23]$.

\subsubsection{Particle evolution equations}

For the simulation of particles, two assumptions about the behavior of particles are made: (i) all particles are rigid spheres with identical diameter $d_{p}$ and density $\rho_{p}$; (ii) only the drag and gravity forces of particles are considered because the ratio of the particle-to-fluid material density is high.

Under the above conditions, the momentum equation of a single particle at position $r$ with velocity $u$ at 
time $t$ can be described using Newton's second law:

$$
\begin{aligned}
\frac{d r_{i}}{d t} & =U_{p, i}, \\
\frac{d U_{p, i}}{d t} & =-\frac{U_{p, i}-U_{g, i}}{\tau_{p}} f+g_{i},
\end{aligned}
$$

where the subscripts $p$ and $g$ represent particle and gas, respectively. The equation shows that the rate of momentum change is equal to the sum of external forces on the particle. The first and second terms on the right-hand side are the drag force between the particle and surrounding fluid and the gravitational force on the particle, respectively. The response time $\tau_{p}$ of a particle with mass $m_{p}$ in the fluid of viscosity $\mu$, based on Stokes flow, is given by

$$
\tau_{p}=\frac{m_{p} C_{c}}{3 \pi d_{p} \mu} .
$$

Clift et al. [6] suggested that for a particle slip-velocity Reynolds number $R e_{p}<200$, which is true for most practical dilute flow systems, the nonlinear correction factor $f$ needs to be added,

$$
f=1+0.15 R e_{p}^{0.687},
$$

where $R e_{p}=\left(\rho_{g}\left|\vec{v}_{p}-\vec{v}_{g}\right| d_{p}\right) / \mu$. Also the Cunningham slip factor $C_{c}$ with mean free path of fluid $\lambda$ is

$$
C_{c}=1+\frac{\lambda}{r_{p}}\left[1.257+0.4 \exp \left(-1.1 \frac{r_{p}}{\lambda}\right)\right],
$$

where $r_{p}$ is the particle diameter.

All three components of the particle momentum are computed using the above equations, but the particles are constrained to the line. The off-line velocity components are used in the PEI models discussed below. Constraining the particles to the line is a limitation of the ODT model.

\subsubsection{Type-I particle model}

During the ODT diffusive advancement, Eq. (15) is solved for the three components of the particle velocity using the local components of the ODT gas velocity in the $x$ and $z$ directions, and zero for the ODT line-directed $(y)$ gas velocity (since $y$ motions are governed by eddy events). That is, dispersion in directions other than the ODT-line direction naturally occur during the diffusive advancement described in Sec. 2.1.1.

The interaction between a particle and an ODT eddy event is defined as both the particle and the triplet map occupying the same space-time. For the Type-I PEI, the particle-eddy interaction is instantaneous in the simulation advancement time $t$. However, to capture the interaction, a finite temporal interval and cubic spatial region of each eddy (of side-length $l$ ) is assumed based on its own time and length scale. The interaction between particles and an eddy evolves in three directions governed by the $x, y$ and $z$ components of the above modified Stokes' law. The interaction is chosen to have the same length scale in all three directions, though other modeling choices could be appropriate. The particle-eddy interaction ends when 

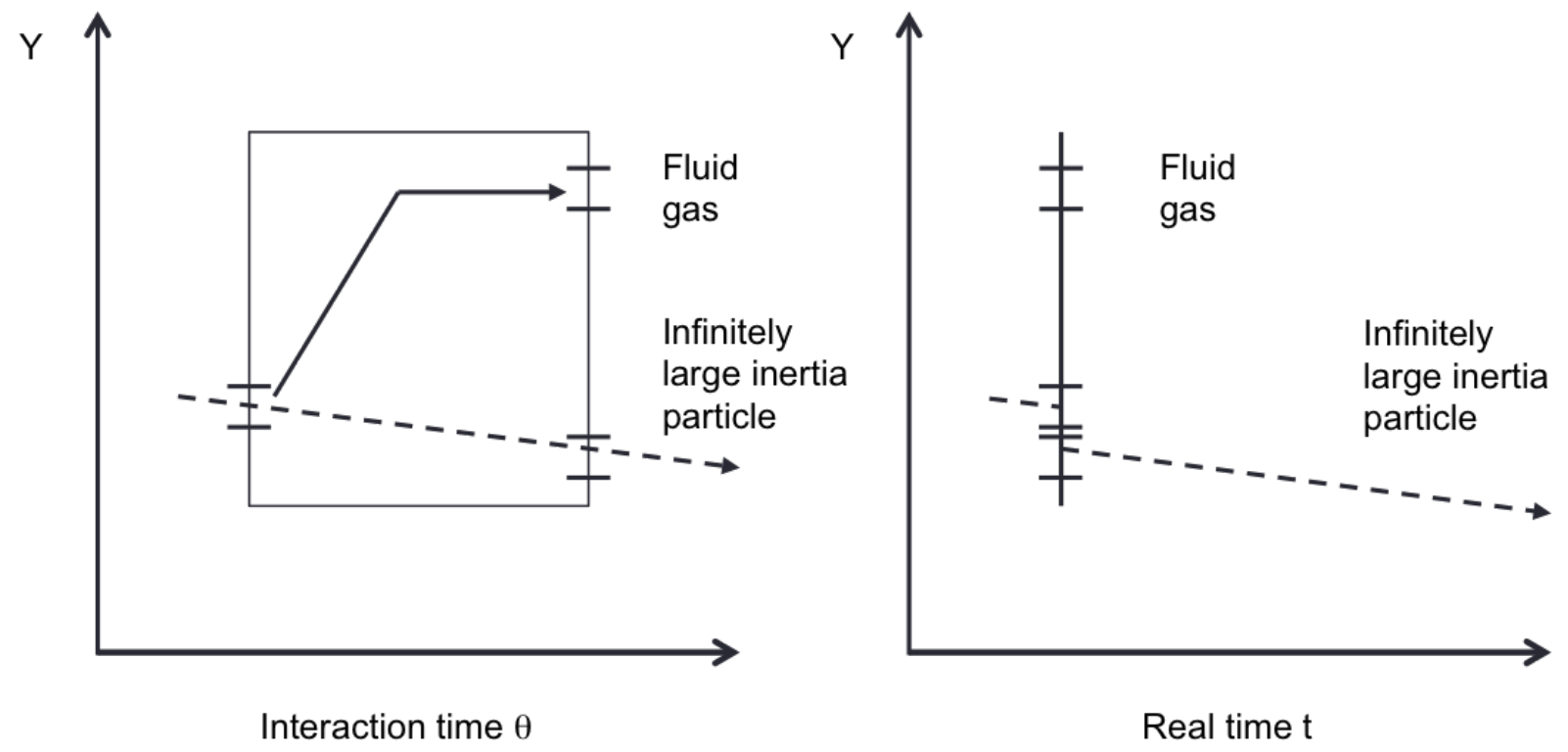

Figure 1: Schematic diagram of the particle-eddy interactions in the interaction and real time coordinates. The figure also illustrates the need to treat the so-called double counting effect. Dashed lines represent the trajectory of a ballistic particle. The rectangular box (left) and vertical line (right) represent eddy events. (Adapted from Schmidt et al. [31])

the particle leaves the idealized eddy or when the eddy lifetime has passed, whichever comes first. The ODT eddy events affect only the line-directed particle velocity and position. However, the particle drag law is solved in all three directions in order to determine the interaction time of a particle with an eddy. A new temporal coordinate is needed which is called the interaction time coordinate, $\theta$, which describes how long the particle interacts with the eddy. Simply speaking, the particle-eddy interaction is instantaneous in real time coordinate $t$ while it exists for finite time in interaction time coordinate $\theta$. Figure 1 shows (among other things) the eddy effect in the interaction time coordinate (left) and real time coordinate (right) in the $y$ direction. When $\theta_{i x n}>t_{e}$, the interaction ceases even if the particles are still in the eddy box, where $\theta_{i x n}$ is interaction time between the particles and eddy. When $\theta_{i x n} \leq t_{e}$, the particles may exit the eddy box by reaching the boundaries of the box.

Eddy velocities in the $x, y$, and $z$ directions are defined to describe the drag force between the particle and the eddy so that particle $y$ positions and velocities after the interaction may be determined,

$$
U_{e}=U_{g},
$$

$$
V_{e}=\frac{\triangle Y_{T M}}{t_{e}},
$$

$$
W_{e}=W_{g} .
$$

Eddy velocities $U_{e}$ and $W_{e}$ are the respective $x$ and $z$ ODT velocity components at the particle location. The eddy velocity $V_{e}$ in the $y$ direction is the turnover velocity of a fluid parcel containing the particles 


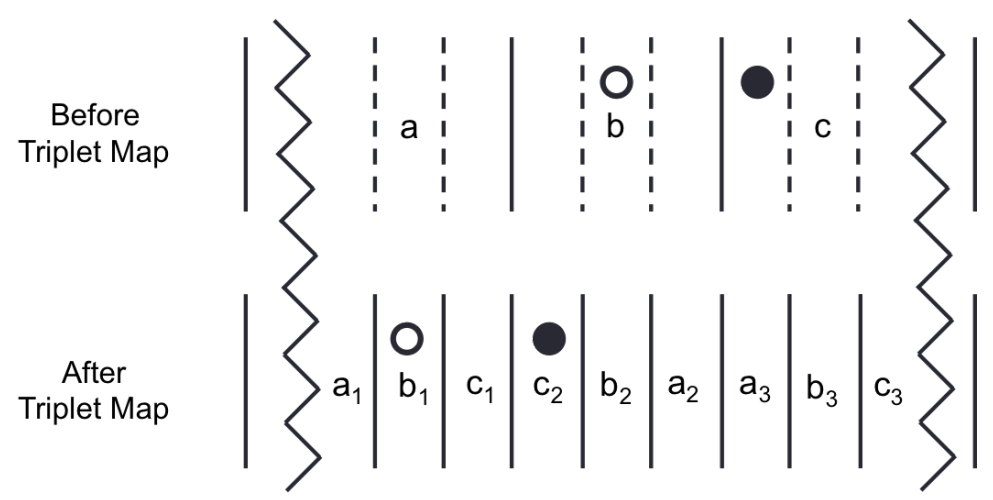

Figure 2: Illustration of the triplet map implementation. Jagged lines indicate eddy edges; solid lines are cell boundaries.

during the triplet map. $\triangle Y_{T M}$ is the displacement of a notional Lagrangian fluid particle by the triplet map at the particle location, as described in the following paragraphs.

As shown in Fig. 2 of the triplet mapping operation, there are three distinct displacements of a given fluid element that correspond to its three subdivisions. Unlike the fluid elements, the particles cannot be subdivided, which requires the determination of which of the three distinct fluid displacements to use in Eq. 20. There are two ways to make this determination. One is to use the discrete implementation of the triplet map (as has been done in some previous ODT implementations) to assign a unique displacement of the fluid that contains the particles. (The present ODT uses a continuous implementation of the triplet map on an adaptive computational grid.) A disadvantage of this discrete approach is that the first and last fluid cells of the eddy subdivisions are not moved and $\triangle Y_{T M}=0$ in Eq. 20. Neglecting small displacement near eddy endpoints has a disproportionate impact near walls, where these small displacements can be the dominant mechanism [28]. This undesired affect could be minimized by using high spatial resolution, but that significantly increases computational costs.

Another more cost-effective approach is a random selection procedure. In the infinitely high-resolution case, all the flow properties including particle distribution are statistically uniform at the fine scale. Any location class of particles is equally distributed among the eddy fluid cells that correspond to three distinct field subdivisions of given fluid element. This indicates that a given particle can be statistically localized to any of the three pre-mapped subdivisions with equal probabilities. Therefore, a random selection of one of the three fluid parcels for the particle environment is used in this work. The procedure is illustrated in Fig. 2 by the open and filled circles that denote notional Lagrangian fluid elements. The notional fluid elements are positioned on the triplet map on the region of the discretized ODT domain. The letters a, b, and c represent the values of fluid profiles in the given cells and serve to identify the cells. After the triplet map, the original profile $(a, b, c)$ becomes $(a, b, c ; c, b, a ; a, b, c)$. The original scalar profile is compressed spatially by a factor of three, and a copy is placed on the first and last third of the eddy domain, whereas the profile is spatially inverted for the middle third. The notional Lagrangian fluid element in a cell with a given fluid 
property (e.g., a, b, or c) will be mapped to a random one of the three post-mapped locations with the same fluid property. This is shown in the figure as the open circle in the cell $b$ is moved to the cell $b_{1}$ (though it could have been cell $b_{2}$ or $b_{3}$ ), whereas the filled circle in cell $c$ is mapped to cell $c_{2}$ (though it could have been cell $c_{1}$ or $c_{3}$ ).

With eddy velocities specified as in Eqs. (19-21), the drag law is integrated to determine the particleeddy interation time. The particle is initially located in the center of the eddy box in the off-line directions, and the eddy box is advected with the $x$ and $z$ eddy-velocity components.

Schmidt et al. [31] found that since the particle transport is implemented instantaneously, but the momentum equation of particles is integrated for the interaction time, the concurrent diffusive advancement would result in a double integration effect. To elaborate this effect, consider a particle that has infinitely large inertia. The particle will not be affected by the eddy. However, as Fig. 1 shows, the double integration effect will produce the shift of the particle velocity and position, which violates physical behavior. To avoid this, the particle velocity $V_{p}^{\text {new }}$ and position $y_{p}^{\text {new }}$ resulting from the particle-eddy interaction are computed by taking the difference of the integration solution of the momentum equation with and without the eddy velocity. This is illustrated schematically in Fig. 3. That is,

$$
\begin{gathered}
V_{p}^{\text {new }}=\triangle V_{p}=V_{p}^{i}\left(\theta_{i x n}\right)-V_{p}^{n}\left(\theta_{i x n}\right), \\
y_{p}^{\text {new }}=y_{p}^{\text {old }}+\triangle y_{p}=y_{p}^{\text {old }}+y_{p}^{i}\left(\theta_{i x n}\right)-y_{p}^{n}\left(\theta_{i x n}\right),
\end{gathered}
$$

where superscript $i$ and $n$ indicate with and without the effect of the eddy, respectively. The result is a particle-eddy interaction with the expected behavior in both the tracer particle limit (particles stay with the fluid) and in the ballistic limit (particles are no displaced by eddy events) avoiding the potential of artificial dispersion suggested in Fig. 1.

\subsubsection{Type-C particle model}

The Type-I PEI model described above leads to an instantaneous displacement and velocity change of the particles at the moment of the occurrence of the triplet map. The Type-C PEI model differs from the Type-I model in that the PEI occurs continuously during the continuous diffusive process. While the eddies occur instantaneously, the effect of the eddies on the particles is implemented over a finite duration during the ODT diffusive advancement. As in the Type-I interaction, each eddy is modeled with a cubical eddy box that exists spatially over the domain $\left[y_{0}, y_{0}+l\right]$ and temporally over the eddy lifetime $t_{e}$. Unlike the Type-I eddy, the interaction is not implemented instantaneously, but rather the eddy velocity is mapped to a spatial-temporal eddy box that starts at and continues after the eddy event. Each eddy box is advected in the off-line directions, and the advection velocity is taken as the average local fluid velocity in the box. The crossing-trajectory effect is captured as the particles move relative to the eddy.

The line-directed eddy velocity is taken as $\pm(2 l / \sqrt{27}) / \beta_{p} \tau_{e}$ based on the root mean square displacement of fluid particles in an eddy due to a triplet map [2], and the sign is randomized. In the eddy space-time 

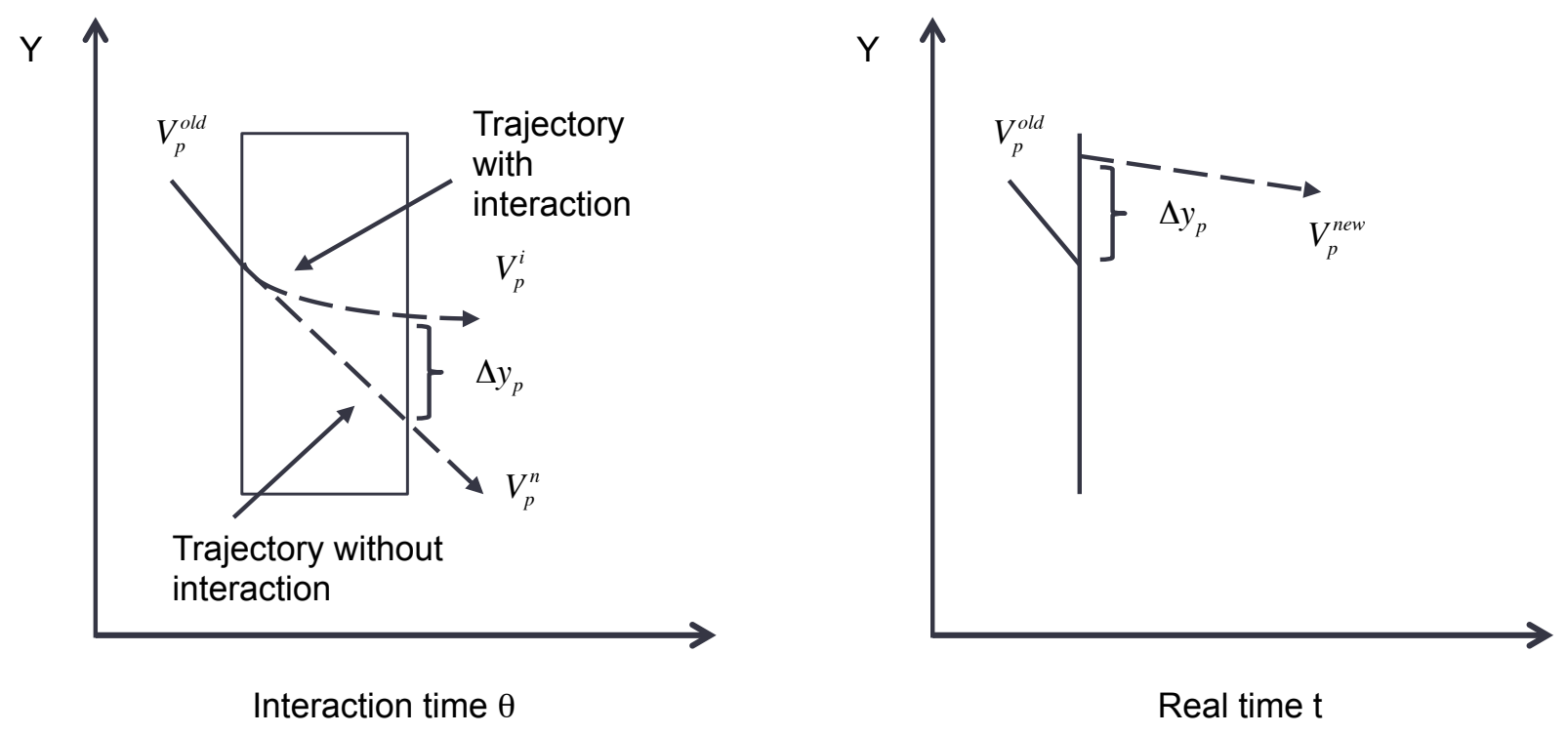

Figure 3: New position and velocity of a particle in the interaction time coordinate (left) and real time coordinate (right) after a Type-I particle-eddy interaction. (Adapted from Schmidt [31].)

map, it often happens that eddy boxes will overlap. In this case, the line-directed velocity component for a given particle consists of the sum of velocities for each eddy box in which the particle is located. The off-line fluid velocities are taken as the local gas velocity on the line.

A significant drawback to the Type-C interaction is that it does not obey the tracer-particle limit. The fluid is mapped instantaneously to new locations during an eddy event, but the particles respond to this fluid motion over a finite time during the diffusive advancement. This may not be statistically important in particle dispersion studies, but in applications such as combustion, where particle temperature-history effects are important, the correct tracer-limiting behavior is important. Another potentially important difference is an apparent time shift. The result of an eddy triplet map is observed at the time of the triplet map in the Type-I eddies while there is a delay of time $\beta_{p} \tau_{e}$ for the same net effect to be observed with the Type-C eddies. The two models are compared further in the next section.

\subsubsection{Instantaneous and continuous particle-eddy interaction}

In this section, the fundamental difference between the Type-I and Type-C particle interaction implemented in this study is discussed. In a Type-I interaction, the particle has an instantaneous displacement in the ODT-aligned direction when it interacts with an eddy. That is, the particle goes through a discontinuous displacement due to the eddy interaction, and then the interaction will expire immediately because the eddy event implementation is instantaneous. Particles interact with a single eddy at a time in the simulation time frame, although the effective eddy lifetimes might overlap. In contrast to the Type-I interactions, there is no instantaneous displacement of particle motion in the Type-C interaction. In the Type-C interaction, 


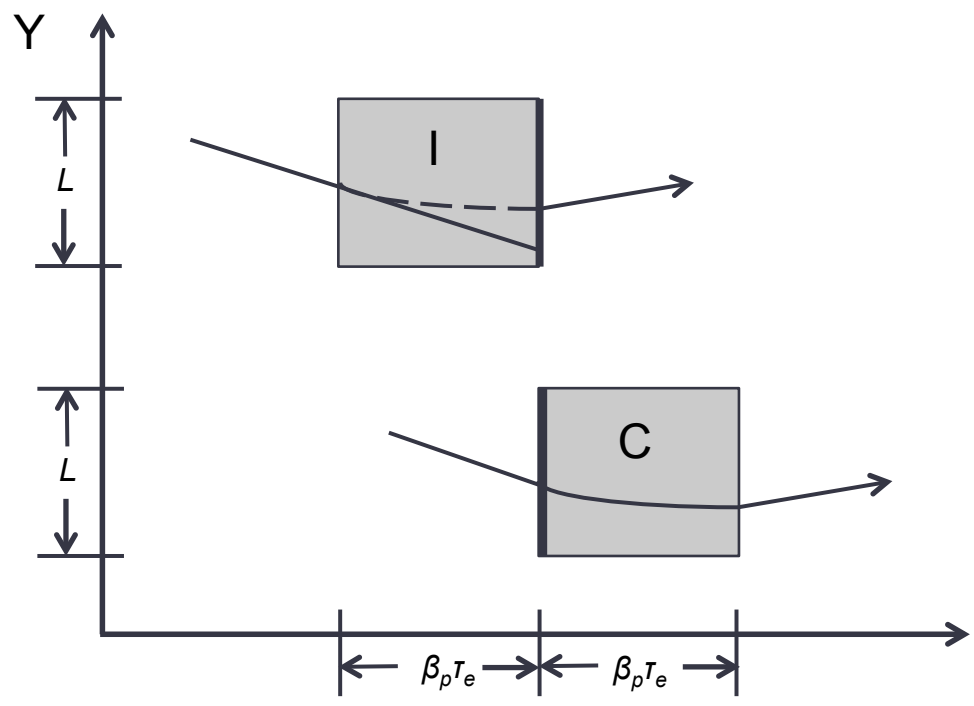

Simulation time

Figure 4: Type-I vs. Type-C particle-eddy interaction. Shadow boxes represent the eddy effect over the spatial domain $\left[y_{0}, y_{0}+L\right]$ and temporal period $\beta_{p} \tau_{e}$; single solid lines represent the particle trajectory; the dashed line represents the particle "interaction" trajectory due to the particle-velocity history in the Type-I interaction [34].

although an eddy event is instantaneous, the eddy effect on particles is allowed to exist in the real time coordinate for the eddy duration. In this sense, the Type-C interaction results in a "delay" in the particle dispersion as Fig. 4 shows. In the Type-C interaction, a particle has continuous interactions with eddies no matter when and where it enters the same space and time region as the eddy has. It is quite likely that one particle can feel the effects of multiple eddies simultaneously. Implementation of the Type-C interactions in ODT requires keeping track of the positions of all eddies from the time each eddy is born until that individual eddy's duration has expired. In the Type-I PEI model, the particles are less likely to interact with the eddy event when the particle-line velocity becomes larger. Assume that the velocity component in the line direction reaches the infinite limit; in that case, there is no way that the particle has a chance to enter the eddy because particle trajectories and the triplet maps are parallel lines in the space-time plane $y-t$. This is not a problem for many typical flows in which the particles move with similar or smaller velocities than the fluid. In contrast, the Type-C PEI model "extends" the eddies in the real time coordinate and thus allows the particles to interact with eddies when they occupy the same spatial-temporal coordinate. The Type-C interaction is advantageous for cases in which particles move very quickly in the line direction. Examples of such flow might include shock-driven turbulence or buoyancy dominated flows where particles may move in a line direction corresponding to strong density gradients driving the mixing process.

A problem with the Type-C model would occur in the case of two-way coupling between the fluid and particle phases for non-passive particles. The instantaneous triplet maps would need to account for the 
influence of the particles on the gas phase. But in the Type-C interaction, the particle motions occur continuously and later than the instantaneous triplet maps that affect the fluid. So there is an inconsistency between the fluid and particle phases that would be difficult to model. Conversely, two-way coupling can be easily done in the Type-I model using kernel functions to account for particle-fluid momentum transfers in a manner similar to the way energy is distributed among velocity components during triplet maps in the current ODT model, (and is the subject of future work).

The particles are able to interact with an eddy in several different ways:

1. The particles could overlap the eddy box in line direction at the time the eddy is born;

2. The particles could enter the eddy box through the offline sides (Type-C only);

3. If the eddy is still active, the particle could re-enter the eddy through the sides of the box (Type-C only).

In the implementation of the Type-C interactions, a new scheme is proposed to allow eddy boxes to move in the $x, y$, and $z$ directions that is similar to the idea of the Type-I interaction in this sense. That is, only the relative motion of the particle and eddy box in all directions is recorded until either the particle crosses out of the box or the eddy lifetime ends. This is very important for the Type-C interaction to accurately capture the effect of crossing trajectories.

The eddy box is advected in the $x$ and $z$ directions using the $x$ and $z$ gas velocity components at the initial particle location for Type-I and Type-C models. Schmidt et al. used the eddy-averaged $\mathrm{x}$ and $\mathrm{z}$ gas velocities for their Type-I simulations $[31,29,30]$. While there is some appeal in using an eddy-average velocity, there are inconsistencies that arise in certain cases. These are most readily observed in the case of tracer particles. Particles that exist in fluid elements with velocities differing from the eddy-average velocity can cross out of the eddy even though they remain associated with fluid elements. This results in a shorter eddy interaction time and less dispersion than that of the actual fluid elements. Naturally, this breaks the coincidence of fluid and tracer particles in the Type-I interactions. This early crossing effect is severe for tracer particles because we find that the parameter $\beta_{p}$ is relatively small leading to significantly reduced tracer dispersion when the eddy-average box velocity is used. It is possible to alter model coefficients to recover the appropriate particle dispersion, but differences will remain between the fluid and tracer evolution, and we find that the dependence of the dispersion on the Stokes number (or particle Froude number) is not correct. For the Type-C eddies, the particles do not match the tracer limit and the sensitivity to the local versus eddy-averaged velocity is less significant. Further, the application of the local velocity is more complicated for the Type-C eddies since it evolves in time. For these reasons, the simpler eddy-averaged velocity is employed for the Type-C interactions, but we recommend the local fluid velocity for Type-I interactions. 

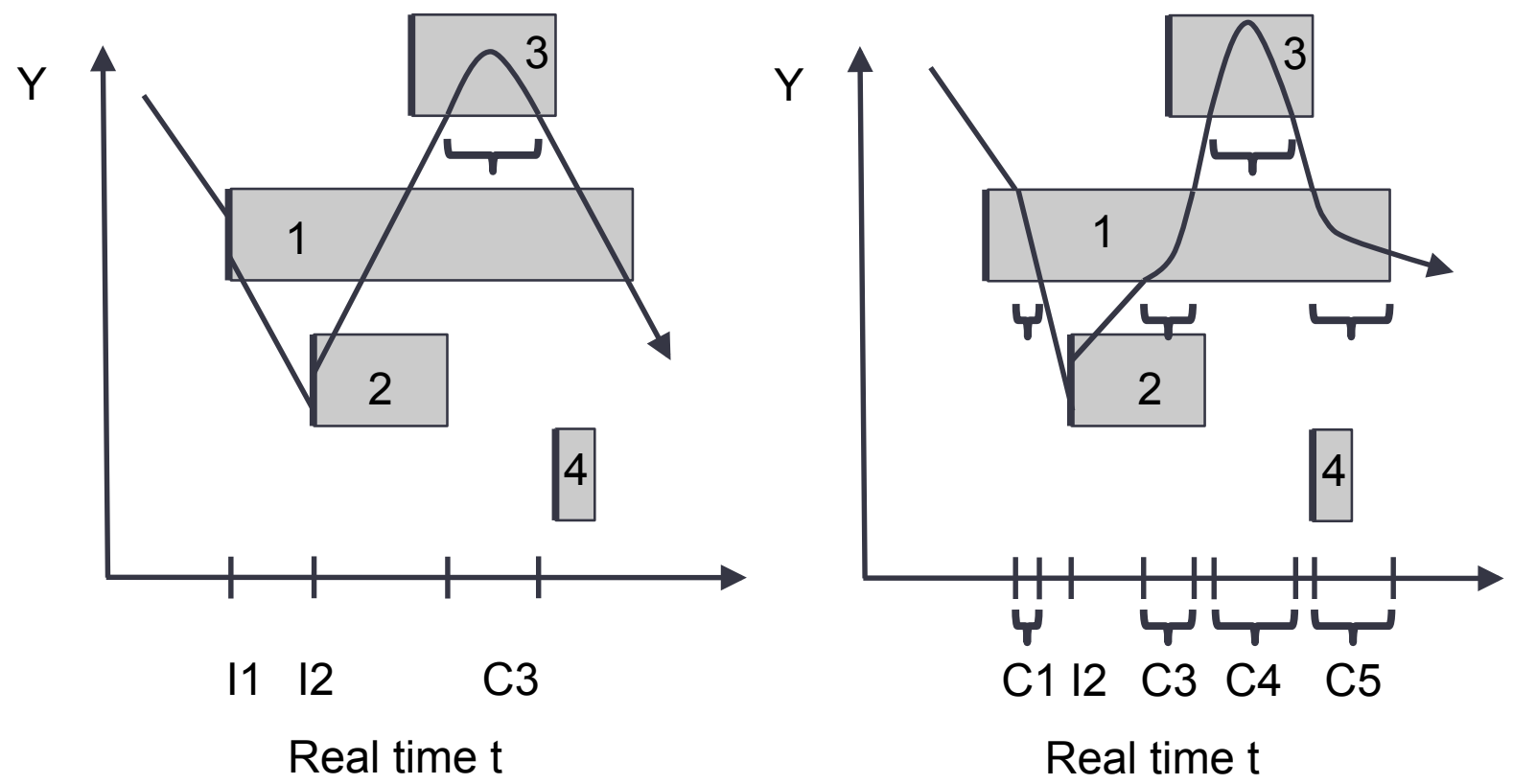

Figure 5: Two illustrative Type-IC particle-eddy interactions.

\subsubsection{Type-IC particle-eddy interaction}

In order to overcome the violation of tracer limit of the Type-C model, another alternative interaction model is introduced here, which is referred to as the Type-IC model. As in Type-C, the eddy is allowed to exist in real time for the duration of the eddy lifetime. However, any particle that overlaps an eddy at the eddy event time undergoes a Type-I interaction, and is not allowed to interact with the same eddy in a continuous Type-C interaction, even if the particle leaves the eddy interaction box and comes back into the box by one of the sides of eddy box. This is because the Type-I interaction already takes into account the entire lifetime of the eddy. Conversely a particle which first enters an eddy box from one of the sides does not undergo a Type-I interaction, but will interact with that eddy in a Type-C manner, and may interact with that same eddy as many times as it (possibly) re-enters the box. It is worth noting that the Type-IC interaction model is able to match the tracer particle limit because in order to have a Type-C interaction a particle must enter an eddy interaction box from either of the sides, and a tracer or gas particle can not do so.

Figure 5 shows two possible particle trajectories in the Type-IC particle-eddy interaction context. In Fig. 5 (left) the particle first interacts with eddies 1 and 2 sequentially in Type-I interactions (I1 and I2). Then it enters eddy 3 through the bottom side of the eddy box and experiences a Type-C interaction $(C 3)$. Although the particle re-enters eddy 1 three times and eddy 2 once, it does not undergo any Type-C interaction with them because the Type-I interaction with eddy 1 and eddy 2 have already been taken into account at the beginning. In Fig. 5 (right) the particle has a Type-C interaction with eddy $1(C 1)$, and 
changes direction in a Type-I interaction with eddy 2 (I2). Then it re-enters eddy 1 (Type-C) (C3), enters eddy 3 (Type-C) (C4), where it changes direction again, and finally enters eddy 1 (Type-C) $(C 5)$.

\section{Turbulent jet configuration}

\subsection{Experiments}

In this study the turbulent dispersion of particles in shear-dominated turbulent flows is studied. Measurements of particle dispersion in round turbulent jets was studied by Kennedy and Moody [15]. These measurements span a range of Reynolds and Stokes numbers, which were obtained by varying the jet velocity, nozzle diameter, and particle diameter. Reynolds numbers based on the jet velocity (air) range from 10000 to 30000. Fully developed turbulent flow conditions at the nozzle exit are used. Hexadecane droplets with number average diameters of 60 and $90 \mu \mathrm{m}$ are used for the study. The mean particle density is $4990 \mathrm{~kg} / \mathrm{m}^{3}$. Monodisperse particles were generated in the experiments with a size uncertainty of $\pm 2 \mu \mathrm{m}$ [15]. The air used in the jet is at room temperature and pressure and thus the particles are essentially non-vaporizing. The particle loading is small with more than 1000 droplet diameters separating particles so that particles do not alter the fluid velocity, nor do they modulate the turbulence; this was verified by the measurements of Kennedy et al [15].

\subsection{Simulations}

The ODT simulations are carried out in a temporally evolving planar jet configuration, which has characteristics similar to those of a spatially evolving round jet [17] and has been routinely applied in ODT simulation, e.g., $[12,8,10]$. The similarity scaling of temporal turbulent planar and spatial round jets is illustrated by constant-density momentum scaling [17]. The width and axial velocity of a spatial round jet evolve as $W \sim x$ and $u \sim 1 / x$, respectively. The scalings for a temporal planar jet are $W \sim \sqrt{t}$ and $u \sim 1 / \sqrt{t}[36]$. These time scalings also follow from the treatment in Schlichting [27, p. 731-2]. If we integrate $d x=u d t$ using $u \sim 1 / \sqrt{t}$ we get $x \sim \sqrt{t}$ so that the $x$ scaling of the temporal planar jet simulated here is the same as the experimental spatial round jet.

To compare the temporal evolution with the spatial experimental measurements, a convective velocity, $U_{m}(t)$, is required to transform the evolution time $(t)$ to the streamwise spatial coordinate $(x)$, which is obtained from the ratio of the momentum flux, $\dot{M}$, to the mass flux, $\dot{m}$,

$$
U_{m}(t)-U_{\infty}=\frac{\dot{M}}{\dot{m}}=\frac{\int_{-\infty}^{\infty} \rho\left(u(y, t)-U_{\infty}\right)^{2} d y}{\int_{-\infty}^{\infty} \rho\left(u(y, t)-U_{\infty}\right) d y},
$$

where $U_{\infty}$ is the axial velocity of the gas phase far from the jet ( $U_{\infty}=0$ in this study) [12]. This assumption implies that all points on the line reach a given measurement plane at the same time. The initial gas velocity conditions for the turbulent planar jet, $U_{g 0}$, are given in Table 1 as a function of the Reynolds number, $R e$, and the jet exit diameter, $D$. The streamwise velocity at the inlet is specified using the following hyperbolic 
Table 1: Initial conditions of gas phase and particle phase $(60,90 \mu \mathrm{m})$, and particle nozzle Stokes number in the $7 \mathrm{~mm}$ jet $\left(S t=\tau_{p} U_{g 0} / D\right)$.

\begin{tabular}{l|c|c|c}
\hline & $R e=10000$ & $R e=20000$ & $R e=30000$ \\
\hline \hline$U_{g 0}$ & $21.5 \mathrm{~m} / \mathrm{s}$ & $43 \mathrm{~m} / \mathrm{s}$ & $64.5 \mathrm{~m} / \mathrm{s}$ \\
$U_{p 0}(60 \mu m)$ & $17.5 \mathrm{~m} / \mathrm{s}$ & $30 \mathrm{~m} / \mathrm{s}$ & $46 \mathrm{~m} / \mathrm{s}$ \\
$S t(60 \mu \mathrm{m})$ & 26 & 53 & 77 \\
$U_{p 0}(90 \mu \mathrm{m})$ & $15 \mathrm{~m} / \mathrm{s}$ & $32 \mathrm{~m} / \mathrm{s}$ & $51.5 \mathrm{~m} / \mathrm{s}$ \\
$S t(90 \mu \mathrm{m})$ & 61 & 122 & 178 \\
\hline
\end{tabular}

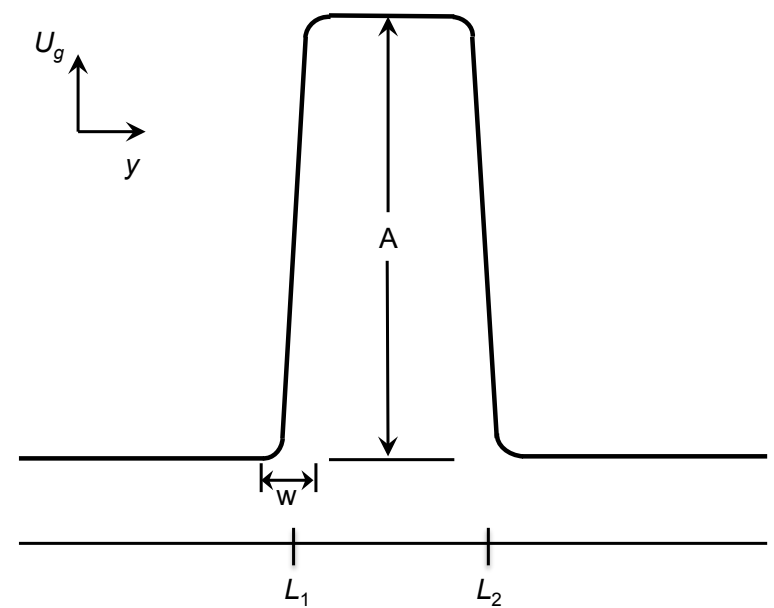

Figure 6: Schematic of the tanh profile used to specify the initial streamwise velocity profile.

tangent function to smoothly transition the velocity in the radial direction and is shown schematically in Fig. 6,

$$
U_{g}(y)=\frac{A}{2}\left[1+\tanh \left(\frac{y-L_{1}}{w}\right)\left(1-\frac{1}{2}\left(1+\tanh \left(\frac{y-L_{2}}{w}\right)\right)\right)\right],
$$

where $A$ is the velocity amplitude, $w$ is the transition boundary layer width, and $L_{1}$ and $L_{2}$ are the middle position of the transitions. Particles with different diameters are injected into the centerline of the jets. The simulation domain width is $40 D$ and the ODT model evolves for $0.11 s$ for all the cases, which is approximately $70 x / D$. The initial temporal resolution is $0.2 \mu s$, the initial spatial resolution is $50 \mu m$, and an adaptive meshing algorithm is used, which refines the mesh as fluctuations cascade to smaller length scales. The initial conditions for the dispersed phase are given in Table 1, in which the initial particle axial velocity, $U_{p 0}$, along the centerline is extrapolated from experimental results. The results reported here are collected over 512 ODT realizations, which are enough to provide stationary ensemble statistics. The ODT parameter values are $C=16, Z=50$ and $\beta_{\text {les }}=0.4$ for all the ODT simulations. 


\section{Results and discussion}

\subsection{Jet evolution}

In order to compare particle results between our ODT results and experimental data, it is first necessary to compare the gas-phase flow characteristics. The ODT-predicted streamwise velocity evolution at the centerline is compared with the experimental measurements [15] in Fig. 7. The mean and fluctuating axial velocities are normalized by the jet exit velocity $U_{g 0}$, and the position is normalized by the jet exit diameter $D$. The decay of the centerline mean and root-mean-square velocities, $U_{c}$ and $U_{c, r m s}$, are typical of free turbulent jets. Overall, the numerical results agree well with experimental data. The ODT mean velocity decays somewhat faster than the experiments at approximately $x / D>30$. The ODT exhibits a Reynolds number similarity and the profiles are very similar for the three simulations, while the measurements exhibit some Reynolds number dependence as shown in the figure. This may be indicative of some differences in the development of turbulence and boundary layers within the jet nozzle; we have not attempted to correct for this in the ODT simulations.

The best fit line through $\log U_{c}$ vs $\log x$ is considered. At $x / D>60$, the ODT gives slopes of $-0.79,-0.95$, and -0.98 for the $\mathrm{Re}=10000,20000$, and 30000 cases, respectively. The results appear to be asymptotic to -1 with increasing Reynolds number. The experimental data are more difficult to fit. There is an obvious "jog" in the $\operatorname{Re}=20000$ data at $x / D=40$, and in the $\operatorname{Re}=30000$ data at $x / D=52$. If we take a fits through the last 4 points in the $\mathrm{Re}=10000$ data and through the points before the "jog" in the other two sets, the slopes are $-0.72,-0.71$, and -0.71 for the $\mathrm{Re}=10000,20000$, and 30000 cases, respectively. For reference, in the region $30<x / D<40$ (corresponding to the slope for the $\mathrm{Re}=20000$ experimental case), the ODT slope ranges from -0.82 to -0.86 for the three cases. We note that the Reynolds numbers studied are not particularly high, and that convergence to a slope of -1 is expected as Re increases.

The ODT velocity fluctuations show the same qualitative trend as the experiments, but the peak that occurs at $x / D \approx 5$ is over-predicted by the ODT by a factor of two. At later times, $x / D>20$, the ODT velocity fluctuations are in good quantitative agreement with the experiments. This is important since most of the particle dispersion (discussed below) occurs at $x / D>30$ in the experiments and simulations.

\subsection{Particle phase}

The results of particle transport are presented in this section, specifically, ODT and experimental results for a turbulent multiphase round jet are compared. A detailed analysis is conducted to assess the performance of the three ODT multiphase interaction models described in Sec. 2.2. In this study, the $\beta_{p}$ value used in the jet flow for all the interaction models is 0.08 .

\subsubsection{Type-I particle-eddy interaction}

The particles have instantaneous displacements during the Type-I interaction with the eddies. The dispersion of particles is predicted for nonzero gravity $\left(g=9.8 \mathrm{~m} / \mathrm{s}^{2}\right)$ as a function of normalized axial 
(a)

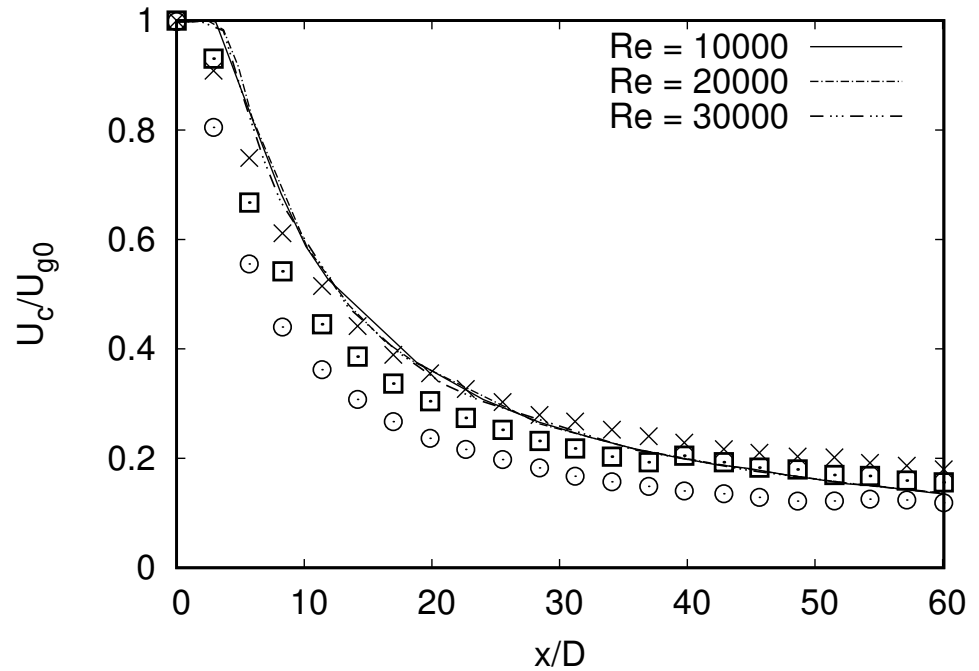

(b)

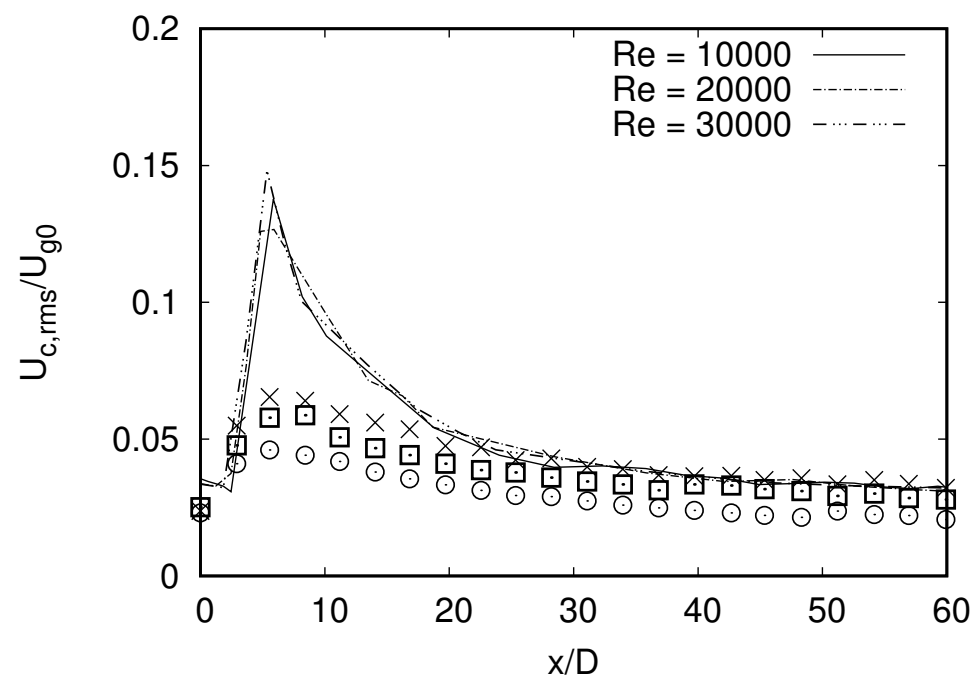

Figure 7: Normalized mean axial velocity (a) and turbulence intensity (b) along the jet centerline. Lines represent ODT predictions. Experimental measurements are represented by cross points $(R e=10000)$, square points $(R e=20000)$, circular points $(R e=30000)$. 
location $x / D$. Figure 8 compares the dispersion data for ODT cases of $60 \mu m$ and $90 \mu m$ particles to experimental measurements for $R e=10000,20000$ and 30000 using the Type-I interaction model. Particle dispersion $D_{p}$ is defined as the root mean square displacement of the particles from the jet centerline, computed using the ensemble of ODT realizations. The particle dispersion increases with the jet evolution. Particles with larger Stokes numbers disperse less and the ODT Type-I interaction model provides good qualitative predictions of this. In the upstream part of jet $(x / D<30)$, the particles are not strongly affected by the fluid flow, due to a lack of large eddy structure. As the large eddies that account for the bulk of the spreading appear later, the particles are transported away from the center of the jet, resulting in non-uniform particle dispersion patterns with particle size.

The particle movement in the jet is strongly influenced by the size of eddies and consequently the response time of the particles. A representative eddy map of the flow field for a single ODT realization is shown in Fig. 9(a), discussed further below.

In Fig. 8, the particle dispersion decreases with increasing Re of the jet. This is true for both the experiments and simulations. We attribute this to the particles having higher $S t$ at higher $R e$, as shown in Table 1. At higher $S t$, the particles are less influenced by the gas fluctuations and disperse less. In addition, at higher Re the exit velocity of the particles is higher (though somewhat lower than the gas exit velocity in all cases). This decreases the particle residence time in the flow. The higher $S t$ at higher $R e$ also contributes to the crossing-trajectory effect, which decreases dispersion, when particles exit an eddy prior to the eddy lifetime $[34,38,32]$.

As the $R e$ and thus $S t$ increase, Fig. 8 shows that the relative differences between the dispersion of the 60 and $90 \mu \mathrm{m}$ particles decreases. This is true for both the experiments and the simulations. For the ODT, at $x / D=50$, the difference in dispersion of the 60 and $90 \mu \mathrm{m}$ particles is approximately 180,155, and $90 \mathrm{~mm}^{2}$ for $R e=10000, R e=20000$, and $R e=30000$, respectively. The relative difference in the Stokes numbers for the two particle sizes are nearly the same for the three Reynolds numbers (as shown in Table 1), nevertheless, the difference between the dispersion of the two particle sizes decreases as $R e$ increases. This is due to the increase in the magnitude of the Stokes numbers, which decreases the magnitude of the dispersion, as noted above, an effect previously documented with ODT particle modeling [34]. In the previous subsection, the power law scaling of the velocity with $x / D$ was observed to approach similarity differently with increasing Reynolds numbers when comparing the measurements and the ODT predictions; the relative differences in dispersion observed here are consistent with those differences in the velocity evolution.

Figure 10 shows the mean axial particle velocity along the centerline for the two particle sizes in the three different $R e$ jets at different axial positions. Overall, there is a good agreement between numerical and experimental results. Initially the particles are injected at a lower velocity than the fluid. At the nozzle exit the particles tend to accelerate to catch up to the air, and then their velocity decreases due to momentum exchange as the particles relax to the decaying gas velocity. The differences between the ODT 


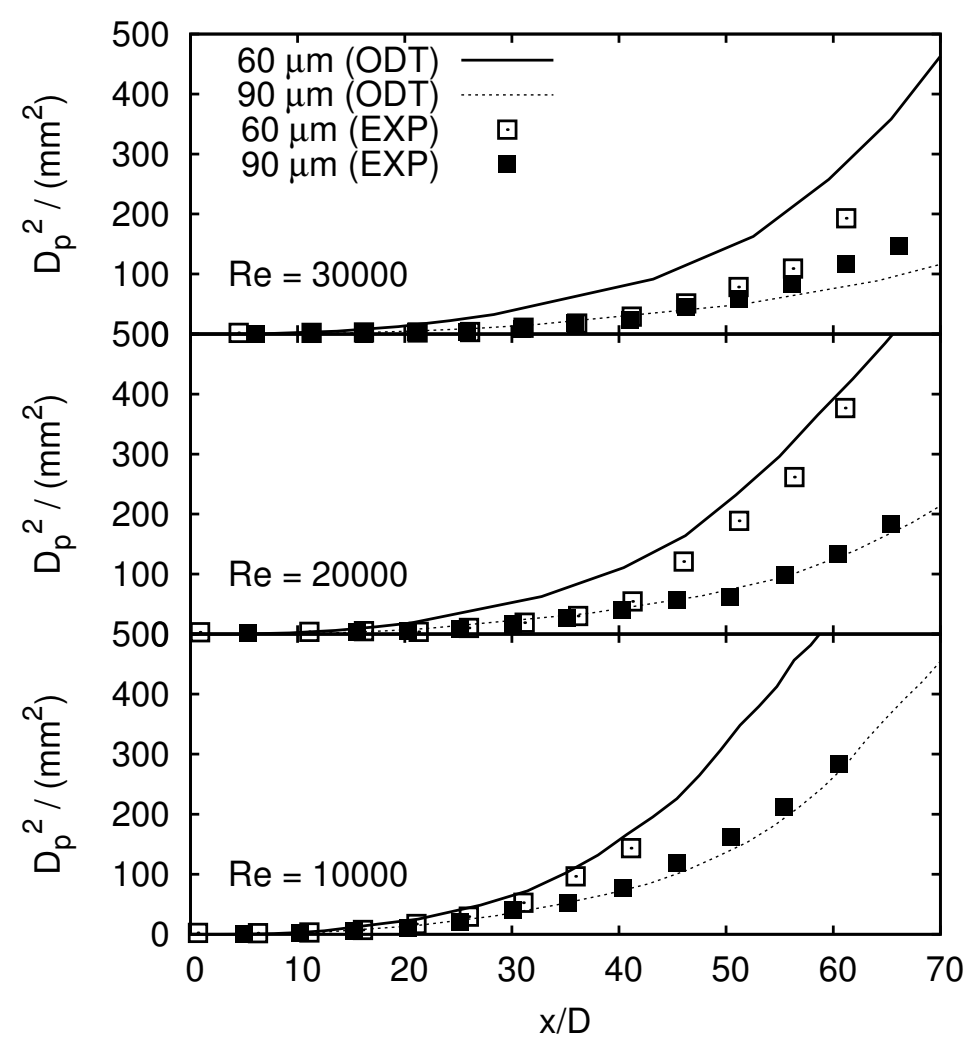

Figure 8: Type-I dispersion of $60 \mu \mathrm{m}$ and $90 \mu \mathrm{m}$ particles in the $7 \mathrm{~mm}$ jet with $R e=10000,20000$ and 30000 . 
(a)

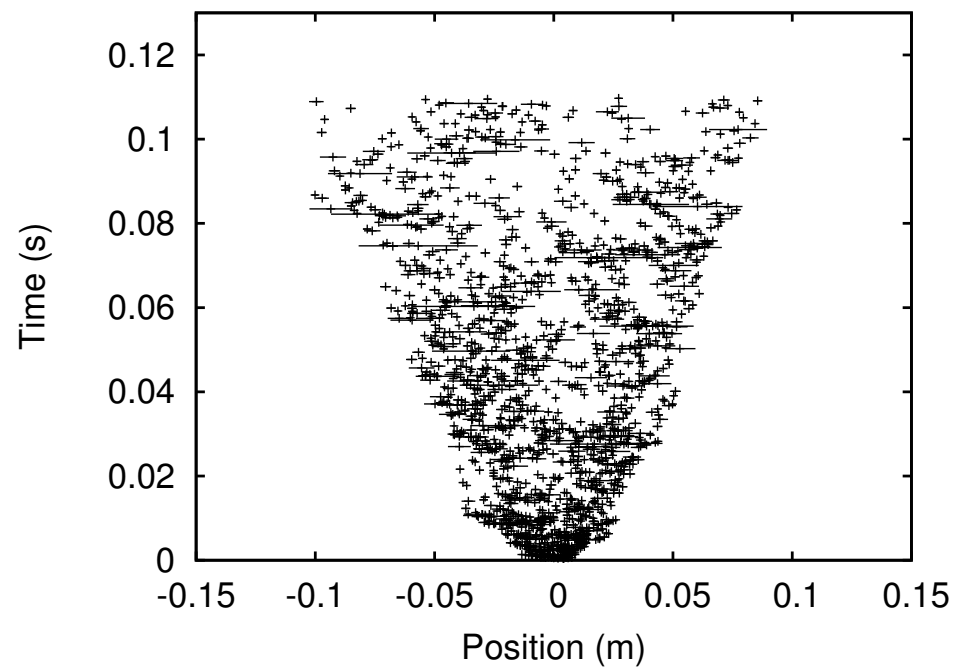

(b)

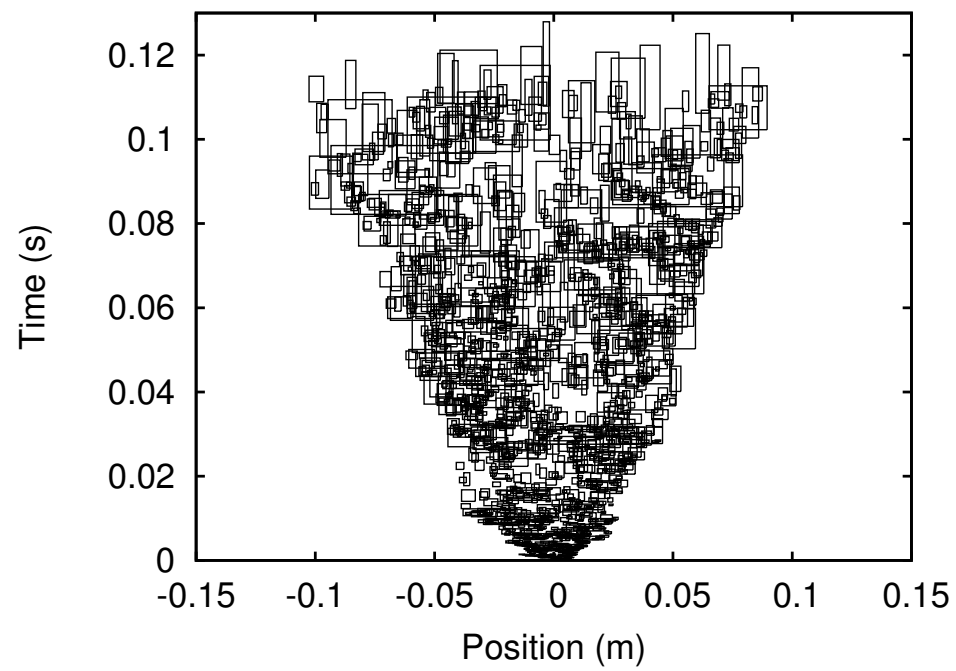

Figure 9: Maps of eddy sizes, locations, and occurrence times for representative ODT realizations for jet flow. Plot (a) shows instantaneous eddy locations for a Type-I interaction; plot (b) shows eddy "box" extents for a Type-C interaction. 


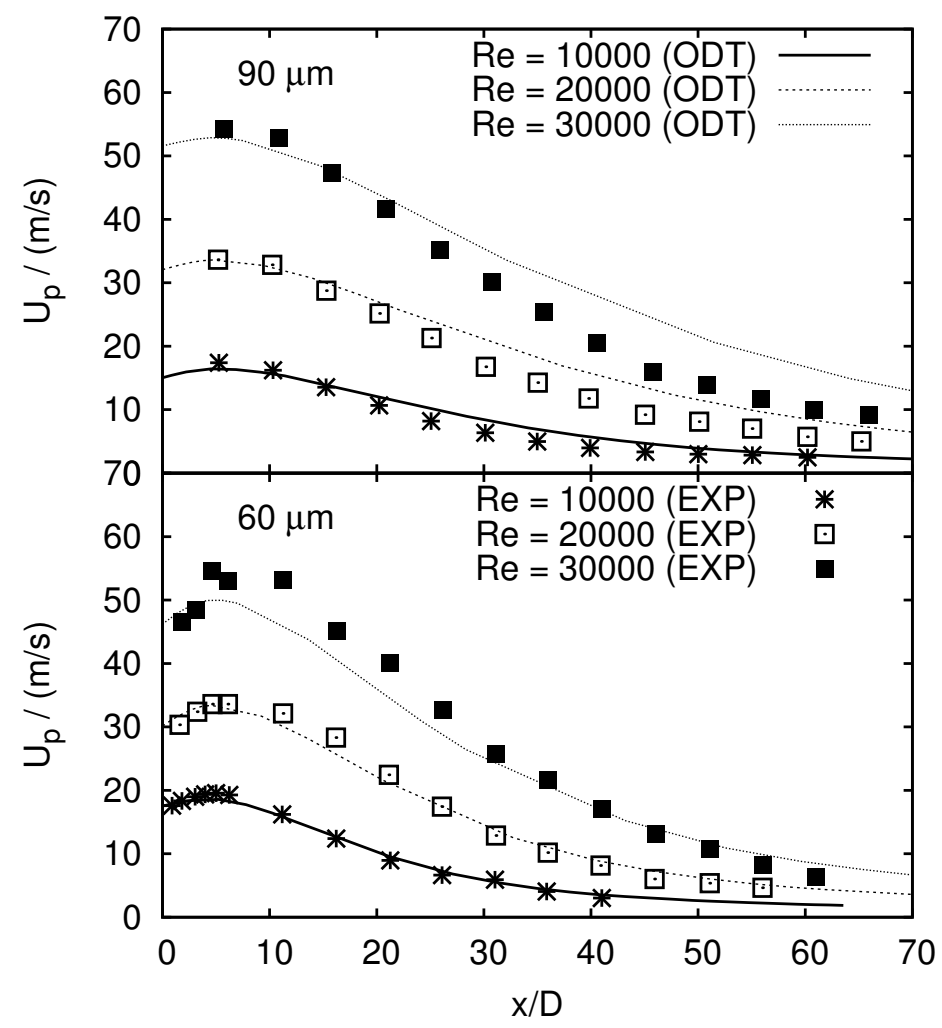

Figure 10: Type-I mean streamwise velocities of $60 \mu \mathrm{m}$ and $90 \mu \mathrm{m}$ particles in the $7 \mathrm{~mm}$ jet.

\subsubsection{Type-C particle-eddy interaction}

In the Type-C model, the eddy events are instantaneous, but the particle-eddy interaction is continuous, with particles influenced by the eddy during the ODT diffusive advancement in the flow evolution coordinate. 


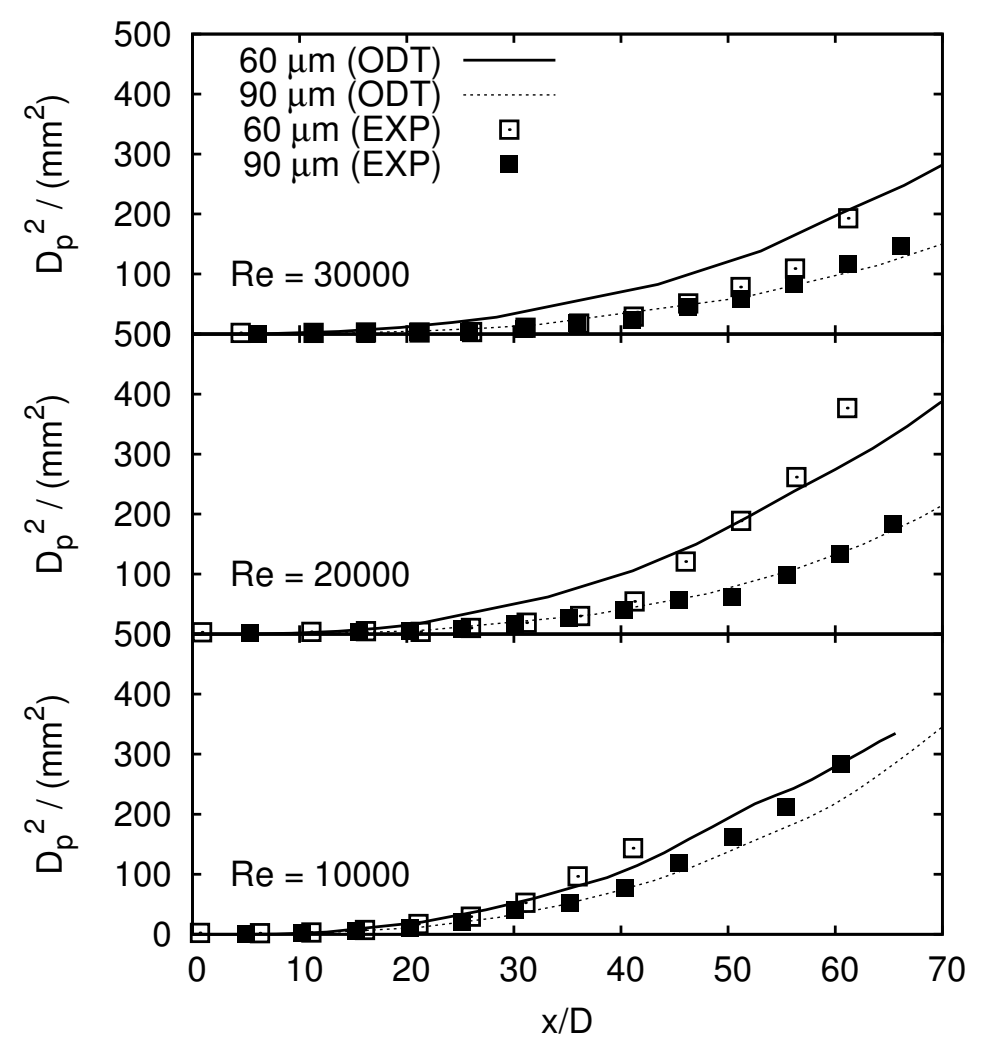

Figure 11: Type-C dispersion of $60 \mu \mathrm{m}$ and $90 \mu \mathrm{m}$ particles in the $7 \mathrm{~mm}$ jet with $R e=10000,20000$ and 30000 .

This is illustrated in Fig. 9(b); the overlapping regions of eddy boxes in the figure suggest the possibility of particle interactions with multiple active eddies simultaneously. Figure 11 shows the comparison of the particle dispersion predicted by the Type-C interaction model to the experimental data $R e=10000,20000$, and 30000. In general, the Type-C model is able to predict particle dispersion for this range of Stokes numbers with a similar fidelity as the Type-I model, but there are important differences between the two predictions that will be discussed in the next paragraph. At the highest Reynolds number the agreement with the data appears to be somewhat better than that of the Type-I model. The axial velocities of the particles for the Type-C model for two particle sizes and three Reynolds numbers are shown in Fig. 12, which is similar to Fig. 10.

Here we go beyond the experiment and further examine the Type-C interactions. Figure 13 compares the dispersion of tracer fluid particles to quasi-tracer particle in the case of $R e=30000$. The quasi-tracer particle is defined to have the same properties as a hollow glass particle in previous homogeneous turbulence study [34]. It turns out that the Type-C model underpredicts the tracer limit because of the delayed dispersive 


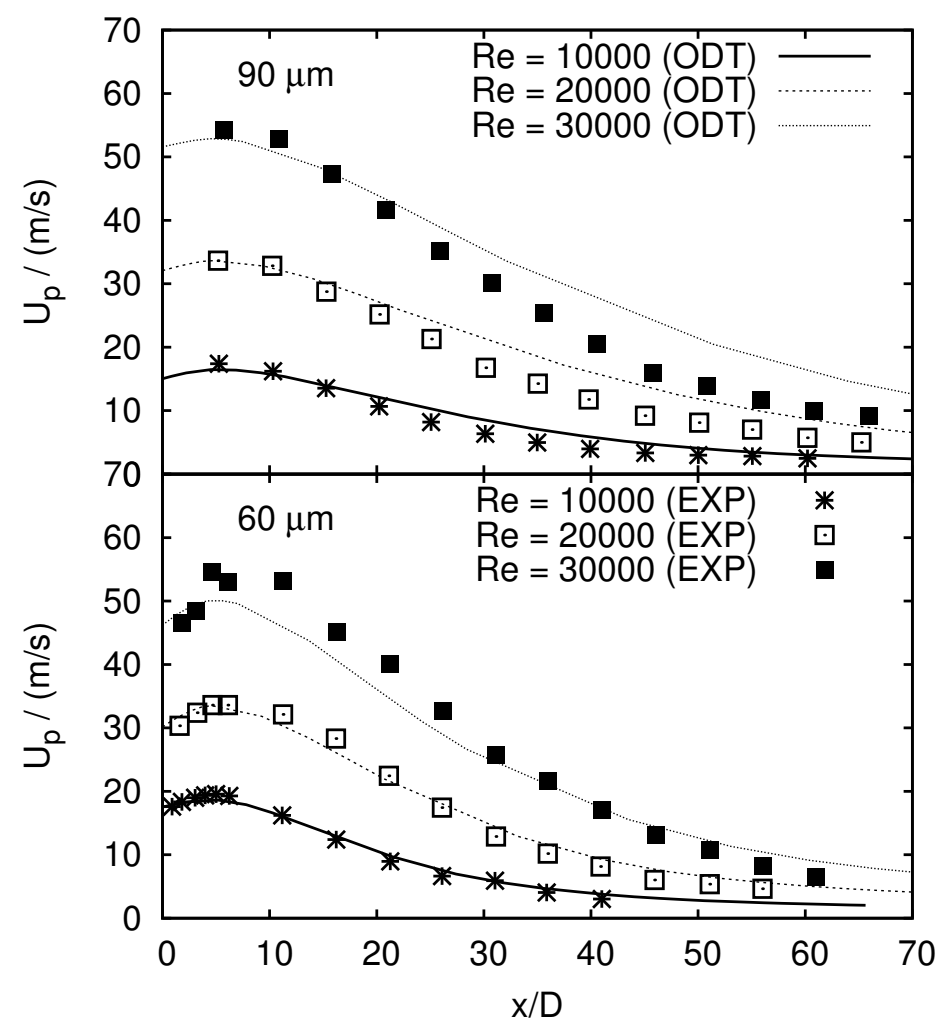

Figure 12: Type-C mean streamwise velocities of $60 \mu \mathrm{m}$ and $90 \mu \mathrm{m}$ particles in the $7 \mathrm{~mm}$ jet. 


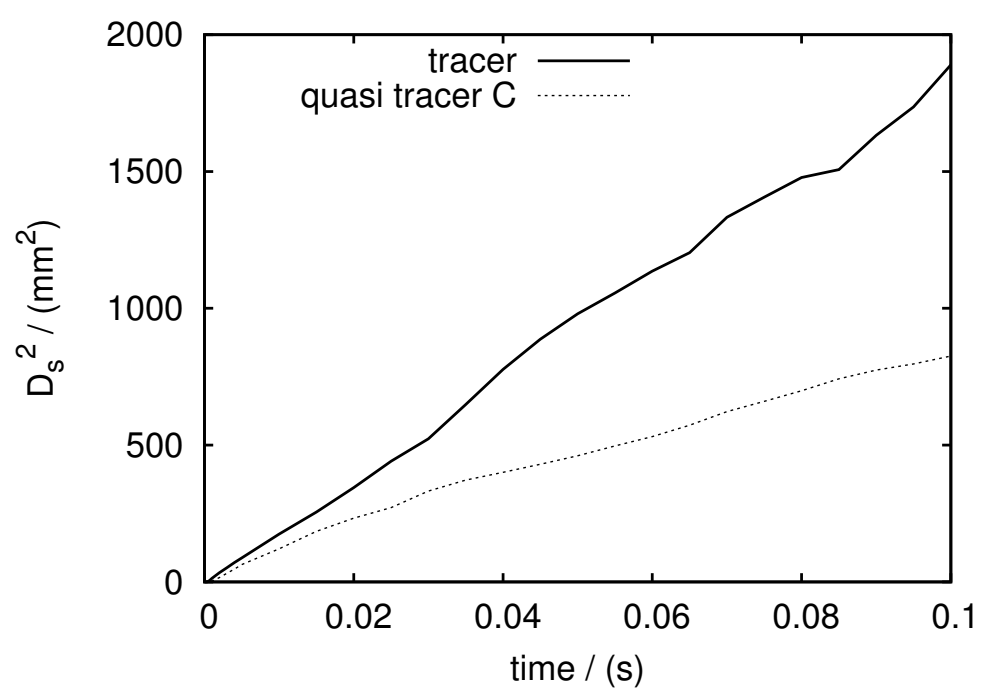

Figure 13: Comparison of Lagrangian dispersion of tracer and quasi-tracer particles predicted by the Type-C model in the $7 \mathrm{~mm}$ jet at $R e=30000$.

motion of small Stokes particles, in contrast to the fluid particles; that is, the fluid particles are displaced at the eddy-occurrence time and the Type-C quasi-tracer particles undergo the same displacement over an eddy lifetime. Since the greatest dispersion is associated with the largest and longest lifetime eddies, this is not a trivial difference. Figure 14 shows the comparison of the Type-I and Type-C interaction models for the dispersion of $60 \mu \mathrm{m}$ and $90 \mu \mathrm{m}$ particles in the $R e=10000$ jet. The Type-I model gives higher dispersion than the Type-C model because, in the Type-I model, the full PEI occurs at the occurrence of the eddy, thus enabling the particles to move earlier. This is consistent with Fig. 4. The large Stokes-number particles tend to retain their velocities longer, and therefore their dispersions are more independent of the PEI type during the early stage of the jet evolution in which the eddy time scales $\tau_{e}$ are small. With the increase of $\tau_{e}$ to the order of magnitude of the inertial response time of large particles, the large particles begin to show different dispersive behaviors for the two different interaction models. In contrast, the small particles adjust to the local jet velocities more quickly, leading to significantly different dispersions between the two interaction models much earlier in the jet.

Quasi-tracer dispersion in the Type-I and Type-C models is further illustrated by considering particle number density profiles. Simulations were performed in the $\mathrm{Re}=20000,7 \mathrm{~mm}$ jet using 1000 hollow glass particles uniformly distributed across the domain. 2000 realizations were computed and the number density distribution evaluated using 101 uniformly spaced bins. Figure 15 shows the results for Type-I and Type-C models at five downstream locations. The profiles are shifted vertically for clarity of presentation. There is some statistical noise in the profiles, but the differences between the Type-I and Type-C models are clear. The Type-I model shows a nearly uniform profile, which is consistent with the continuity constraint for 


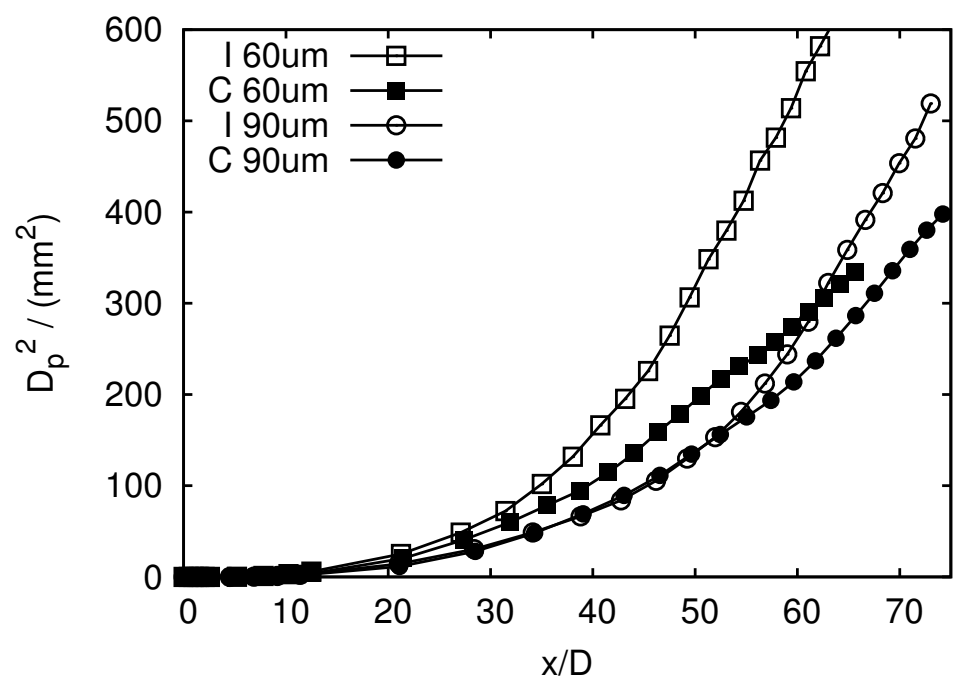

Figure 14: Comparison between the dispersion of $60 \mu \mathrm{m}$ and $90 \mu \mathrm{m}$ particles in the $7 \mathrm{~mm}$ jet for $R e=10000$ predicted by the Type-I and Type-C models.

constant density flows. (The Type-IC model, not shown, behaves like the Type-I model.) Conversely, the Type-C model has a trimodal distribution and does not obey the continuity constraint. In the Type-C model, the number density is depressed in high shear regions of the jet; particles are transported from the high shear region outward and inward towards the jet center, resulting in three peaks in the number density profile. Effectively, the local particle dispersivity in the Type-C model is accentuated in the shear regions since the particles feel the effects of eddies that occurred at earlier times, while the jet turbulence intensity decreases with time. This results in a mean drift of particles out of the shear regions.

Such particle drift is also known to occur in stochastic particle dispersion models based on measures of the turbulence properties as is commonly employed in both RANS and LES simulations for Lagrangian particles. MacInnes and Bracco observed this uneven particle dispersion in mixing layers and jets in the tracer limit using discrete and continuous random walk models (DRW and CRW) [22]. Normalized number density profiles there showed severe deviation in the particle density for the DRW and CRW models, peaking greater than three and five times the value expected based on continuity for the CRW and DRW models, respectively, in the jet configuration. Such model errors were attributed to gradients in the fluctuating fluid velocities. This effect has also been investigated in the context of boundary layer transport of particles as discussed in Iliopoulos and Hanratty [14]. Corrections have been developed to reduce this effect that take advantage of knowledge of the average fluctuation velocities $[22,14]$.

The fluctuations in the Type-C particle density appear to occur due to the variation in the stream-wise velocity fluctuation profiles and are related to the delay in the particle dispersion. That is, particle dispersion occurs starting at the eddy event and is not completed until after the eddy lifetime. In developing flows 


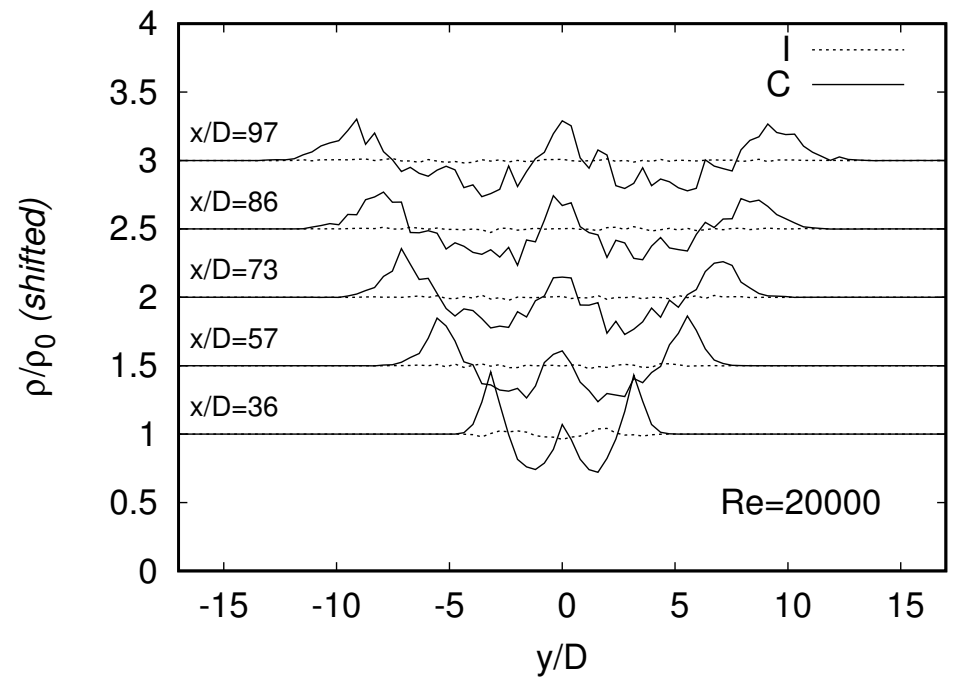

Figure 15: Particle number density profiles for the Re=20000, $7 \mathrm{~mm}$ jet at five streamwise locations. The particle number density is normalized by the freestream number density.

this leads to small inconsistencies in the particle and fluid dispersion for Type-C particles. In the Type-C approach within ODT, the uneven particle dispersion is less significant than observed in, e.g. [22], possibly due to the difference in the magnitude of stream-wise versus cross-stream gradients. Still, the fact that Type-C interactions do not inherently match fluid continuity is a reason to prefer the Type-I model in cases where the particles largely follow the fluid motion.

\subsubsection{Type-IC particle-eddy interaction}

As described in Sec. 2.2, the Type-IC model is considered to be the most robust PEI model in that it not only allows the particles to interact with multiple eddies at the same time but also matches the tracer limit. Figure 16 shows the comparison between experimental and simulation values of particle dispersion in the $7 \mathrm{~mm}$ jet using the Type-IC model that reproduces the experimental results. The prediction of particle axial velocities by the Type-IC model will not be shown here because its comparison to experimental measurements is within $5 \%$ difference of the results of the Type-I model, shown in Fig. 10. The similarity between the Type-I and Type-IC models is due to the relatively low line-directed particle velocity. The line directed particle velocity is due to the turbulent advection, precluding strong transverse eddy trajectory crossing effects. The tracer dispersion is also well predicted by the Type-IC model with the combined instantaneous and continuous interactions. This is shown in Fig. 17, where the comparison of the radial dispersion of quasi-tracer particles and tracer particles in the $7 \mathrm{~mm}$ jet for $R e=30000$ are plotted. This comparison shows the correct model behavior. 


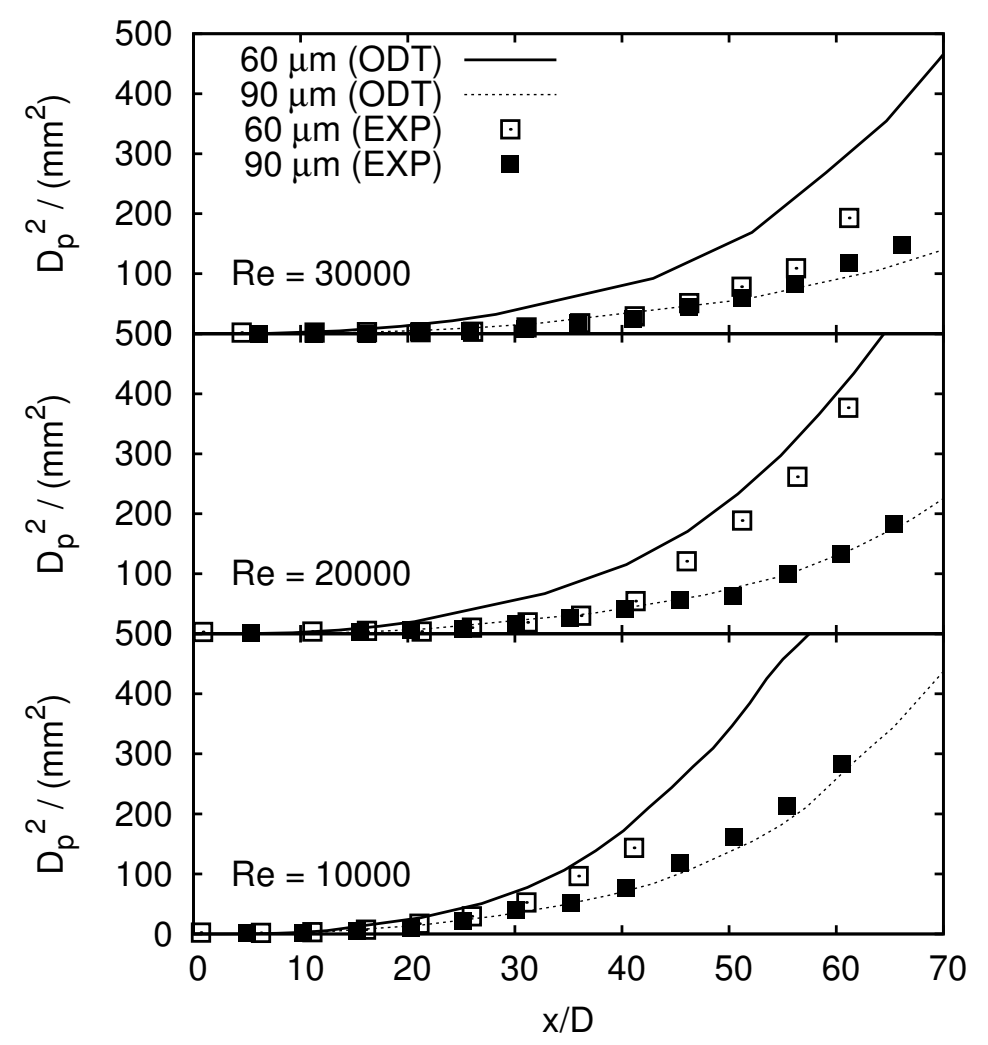

Figure 16: Type-IC dispersion of $60 \mu \mathrm{m}$ and $90 \mu \mathrm{m}$ particles in the $7 \mathrm{~mm}$ jet with $R e=10000,20000$ and $R e=30000$. 


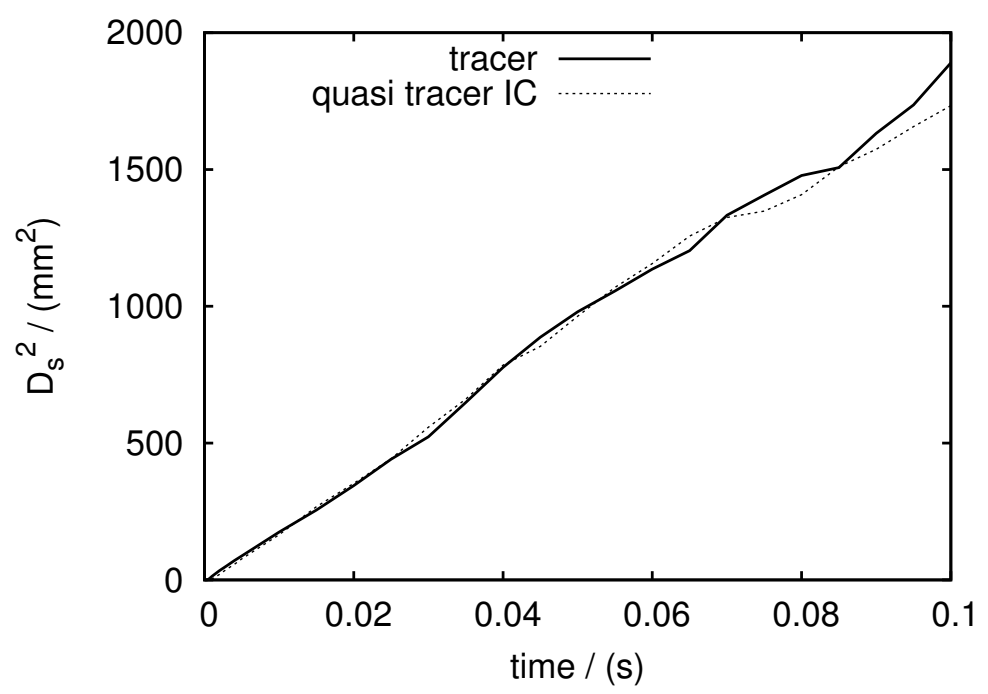

Figure 17: Comparison of Lagrangian dispersion of tracer and quasi-tracer particles predicted by the Type-IC model in the $7 \mathrm{~mm}$ jet at $R e=30000$.

\subsubsection{Lagrangian dispersion}

The ODT simulation results for particle dispersion predicted by the three PEI models are presented in Lagrangian form in Fig. 18. The ODT data are presented as dispersion statistics in the simulated ODT time coordinate. Just as we converted the temporal ODT to the spatial domain for comparison above, the experimental data was converted to the temporal domain in [15] as their quasi-Lagrangian results, which were computed using the "average time-of-flight" of the particles to each measurement plane. (Those results compared favorably with the "true" Lagrangian statistics that were also computed in [15].) Note that this average time-of-flight to each measurement plane will depend on the local particle dispersion due to radial velocity variation. This differs somewhat from the ODT treatment in that the implied time to reach a given measurement plane in ODT is uniform on the line, as noted above. A spatial, cylindrical ODT model would not have this limitation (though particles would still be constrained to the line). Similar to the previous Eulerian predictions, small particles respond to the fluid quickly and approach the fluid velocity in shorter times than large particles, thereby dispersing faster in the jet at a given Re. The Type-I and Type-IC models are very similar, and give reasonable predictions. The results and comparisons of the model and experiments are similar to those presented earlier, especially in terms of the trends. At early times, there is some over prediction of the data, especially for the high Re $60 \mu \mathrm{m}$ cases, but this is more obvious on the log scale presented where the dispersion is very low.

Lagrangian particle dispersivity, $\mathcal{D}_{L}$, can be defined as

$$
\mathcal{D}_{L}=\frac{1}{2} \frac{d D_{p}^{2}}{d t}=\frac{1}{2} \frac{d}{d t}\left\langle D_{p}(t) D_{p}(t)\right\rangle,
$$



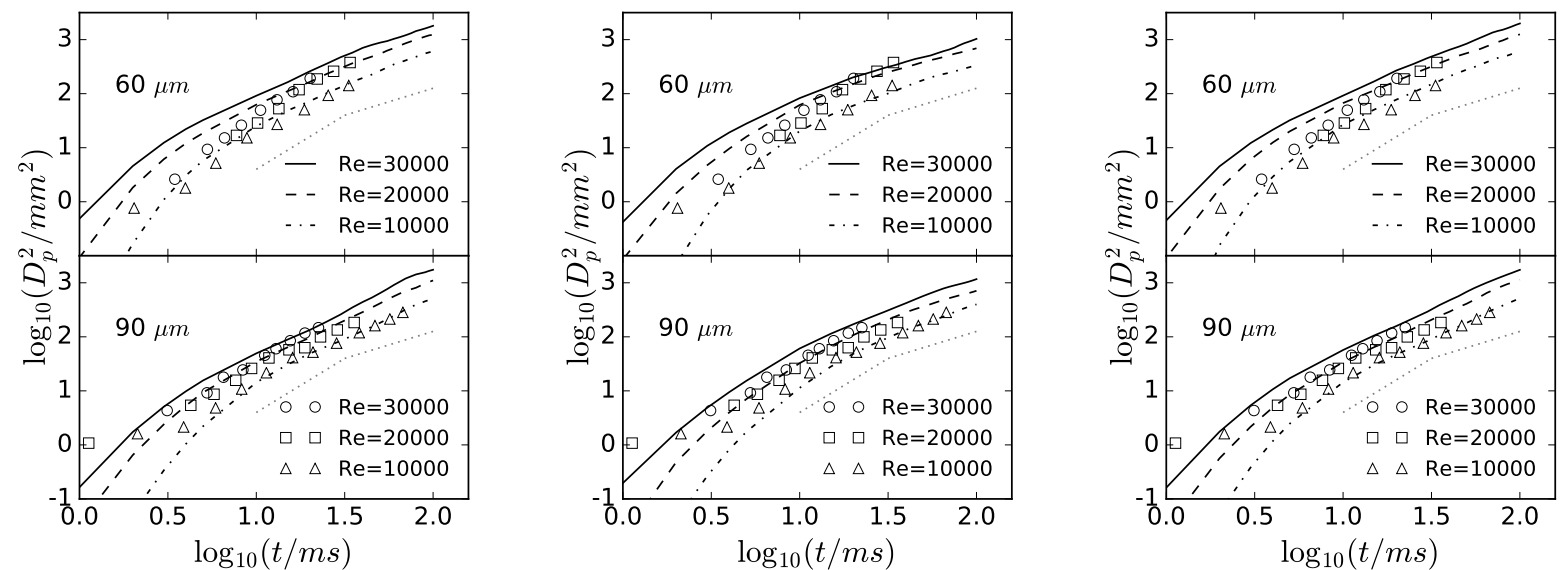

Figure 18: Lagrangian dispersion of $60 \mu \mathrm{m}$ and $90 \mu \mathrm{m}$ particles predicted by the three model types in the $7 \mathrm{~mm}$ jet. The dotted lines show slopes of 2 and 1 for reference.

The particle dispersivity is estimated in the linear portion of the Lagrangian dispersion curves by using a least-squares fit. Table 2 compares ODT simulation results of $\mathcal{D}_{L}$ to the values reported in Kennedy's study [15] at $R e=20000$ and 30000. $\mathcal{D}_{L}$ increases with increasing Reynolds number. Taylor's theory [35] shows that the mean-square dispersion of fluid particles in stationary homogeneous turbulence is a quadratic function of evolution time and behaves linearly with time for long times-of-flight. Batchelor [3] analyzed the transport of fluid particles in shear flow and showed that the dispersion increases linearly with time, and the dispersivity keeps constant. However, a larger Stokes-number particle is not expected to have the behavior of a fluid particle due to its finite inertia. As discussed before, the Stokes number of inertial particles determines how they respond to the fluctuations of the surrounding flows. The particle would eventually tend to respond to all the velocity fluctuations of the gas phase when the local particle Stokes number is $O(1)$.

Figure 18 suggests that an approximately linear region after about 20 and 30 milliseconds, respectively, where they achieve a Stokes number of $O(1)$. The figure includes dotted lines with slopes of 2 and 1 on the $\log$ scale, which indicate the same power law scaling exponents. Table 3 presents the long time exponents for the experimental data and the ODT simulations. These were computed by fitting a line through the last three measurement points of the $\log (t)$ and $\log \left(D_{p}^{2}\right)$ data. The values for the experimental data vary between 1.5 and 2.1, which the ODT are closer to 1 . The Type-I and Type-IC values are similar, as expected, while the Type- $\mathrm{C}$ values tend to be somewhat lower. Due to time-space transformation used as described above, the scaling exponents of the ODT in the spatial coordinate will follow from those presented here in the temporal coordinate through the relation $x \sim \sqrt{t}$. That is $D_{p}^{2} \sim x^{2}$. 
Table 2: Particle dispersivity $\mathcal{D}_{L}\left(d_{p}=60 \mu \mathrm{m}\right.$ and $\left.90 \mu \mathrm{m}\right)$ in the $7 \mathrm{~mm}$ jet.

\begin{tabular}{c|c|c|c|c|c}
\hline$d_{p}$ & Re & Exp & Type-I & Type-C & Type-IC \\
\hline \hline $60 \mu m$ & 20000 & 0.0079 & 0.0067 & 0.0039 & 0.0069 \\
& 30000 & 0.010 & 0.0095 & 0.0053 & 0.0111 \\
$90 \mu m$ & 20000 & & 0.0066 & 0.0037 & 0.0068 \\
& 30000 & & 0.0106 & 0.0063 & 0.0103 \\
\hline
\end{tabular}

Table 3: Power law exponents at late times using the data of Fig 18.

\begin{tabular}{c|c|c|c|c|c}
\hline$d_{p}$ & Re & Exp & Type-I & Type-C & Type-IC \\
\hline \hline $60 \mu m$ & 10000 & 1.8 & 1.0 & 0.87 & 1.2 \\
$60 \mu m$ & 20000 & 1.7 & 0.87 & 0.86 & 1.1 \\
$60 \mu m$ & 30000 & 2.1 & 1.0 & 1.2 & 1.2 \\
\hline $90 \mu m$ & 10000 & 1.5 & 1.3 & 1.0 & 1.3 \\
$90 \mu m$ & 20000 & 1.7 & 1.3 & 0.90 & 1.2 \\
$90 \mu m$ & 30000 & 1.6 & 0.94 & 1.0 & 1.2 \\
\hline
\end{tabular}

\subsection{Parameter sensitivity analysis}

Previous studies, e.g., [12, 21], of parameter sensitivity of ODT parameters $C, Z$, and $\beta_{\text {les }}$ have formed the basis for parameter selection for the jet evolution. In the present work, the fluid phase is not affected by the particles, so we have set the ODT parameters to give reasonable agreement with the fluid evolution, and then focus on the behavior of the particle model, including sensitivity to the $\beta_{p}$ parameter. Variations in the ODT parameters will affect the particle dispersion, but only through the effect on the fluid phase, and reasonable agreement of simulations and experiments is viewed as a prerequisite for analysis of the particle dispersion.

In order to investigate the crossing-trajectory effect of particles in homogeneous turbulence, in previous work we conducted parametric analysis of $\beta_{p}$ that relates the turbulence characteristics to the particle-eddy interaction time [34].

In this section, sensitivity analysis is performed to establish a common basis on which $\beta_{p}$ can be estimated for particle behavior in shear flow among the three PEI models. The analysis is important to guide the future developments and extended applications of the ODT multiphase models. The particle parameter $\beta_{p}$ determines the magnitude of the particle-eddy interaction in the ODT turbulence. High values of $\beta_{p}$ lead to possibly excessive interaction time by increasing the maximum interaction time scale $\beta_{p} \tau_{e}$ and reducing the eddy velocity, $\triangle Y_{T M} /\left(\beta_{p} \tau_{e}\right)$, felt by the particles during interactions. On the other hand, when $\beta_{p}$ is low, the particles interact with "fast" eddies for shorter times. Thus, two competing interaction effects on the particles are controlled by $\beta_{p}$ simultaneously.

Figure 19, 20 and 21 show $\beta_{p}$ sensitivity on the dispersions of $60 \mu \mathrm{m}$ and $90 \mu \mathrm{m}$ particles in the $7 \mathrm{~mm}$ 


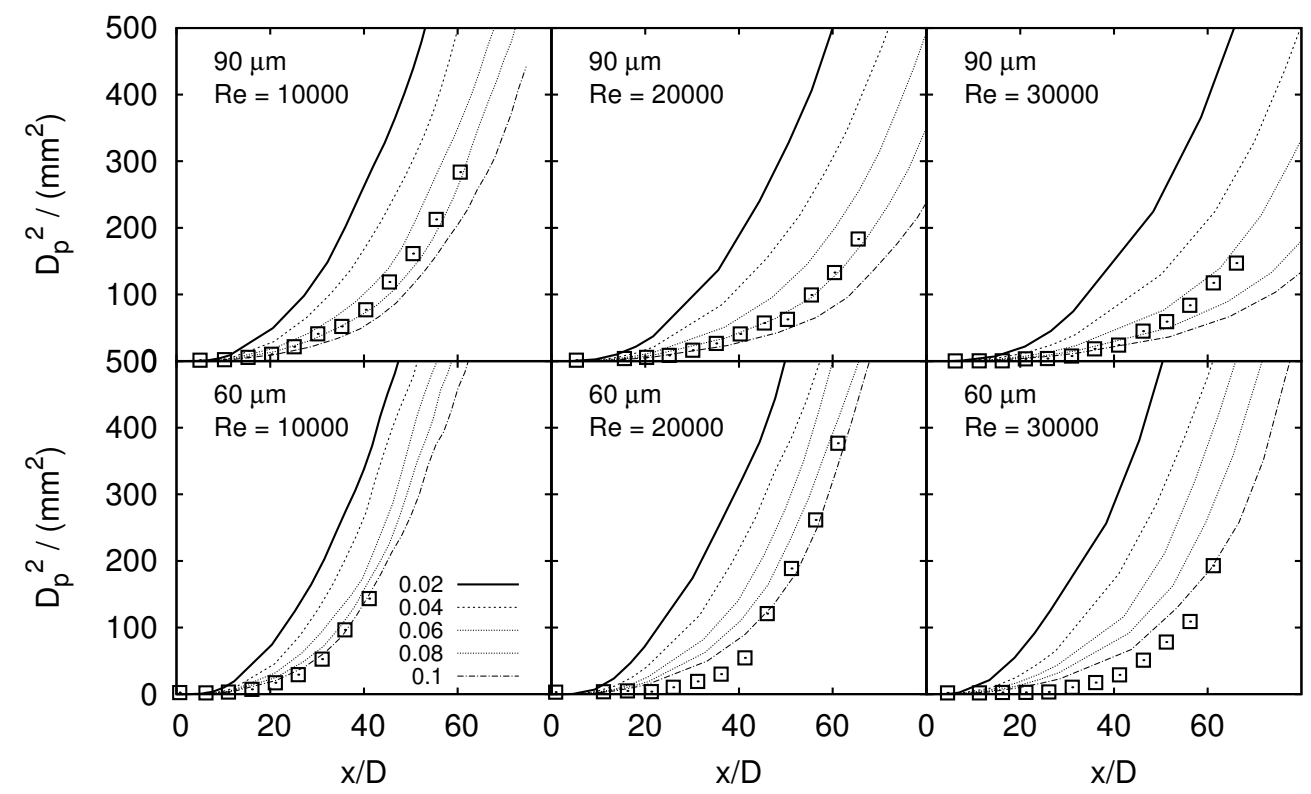

Figure 19: $\beta_{p}$ sensitivity on the Type-I dispersion of $60 \mu \mathrm{m}$ and $90 \mu \mathrm{m}$ particles in the $7 \mathrm{~mm}$ jet with $R e=10000,20000$ and 30000. Square symbols represent experimental measurements.

jet for $R e=10000,20000$ and 30000 predicted by the Type-I, -C and -IC interaction models. Five $\beta_{p}$ values are chosen, that is, $0.02,0.04,0.06,0.08$ and 0.1 , which are similar to those used in the homogeneous turbulence study [34]. Simulations using $\beta_{p}=0.08$ give the best predictions to experimental data.

All eighteen cases show similar particle dispersion sensitivity to $\beta_{p}$ in the shear flow. The particle dispersion decreases as $\beta_{p}$ increases. Increasing $\beta_{p}$ increases the eddy time scale $t_{e}=\beta_{p} \tau_{e}$, making the crossing-trajectory effect more important in limiting the interaction time. As discussed in Sec. 2.2, the interaction time is the lesser of $t_{e}$ and the time to leave the eddy, with this latter time scaling with $l / 2 g \tau_{p}$ under the influence of quasi-steady gravitational settling. Smaller particles with short relaxation times easily adapt to the fluid fluctuations and tend to interact with the eddies for a longer time, so their dispersion is less subject to the crossing-trajectory effect. In contrast to the larger particles, the dispersion of the smaller particles is reduced less with increasing $\beta_{p}$. For a given particle size, the particle dispersions in the high $R e$ case tend to decrease faster than the low $R e$ case when the value of $\beta_{p}$ increases. This is attributed to enhanced trajectory crossing in the higher Re case.

In order to illustrate the $\beta_{p}$ sensitivity in a direct way, a spreading parameter $S_{\beta}$ at given $x / D$ is defined as

$$
S_{\beta}\left(\beta_{p, 1}, \beta_{p, 2}\right)=\left(\frac{D_{p, \beta_{p, 1}}^{2}-D_{p, \beta_{p, 2}}^{2}}{D_{p, \beta_{p, 3}}^{2}}\right) /\left(\frac{\beta_{p, 2}-\beta_{p, 1}}{\beta_{p, 3}}\right),
$$




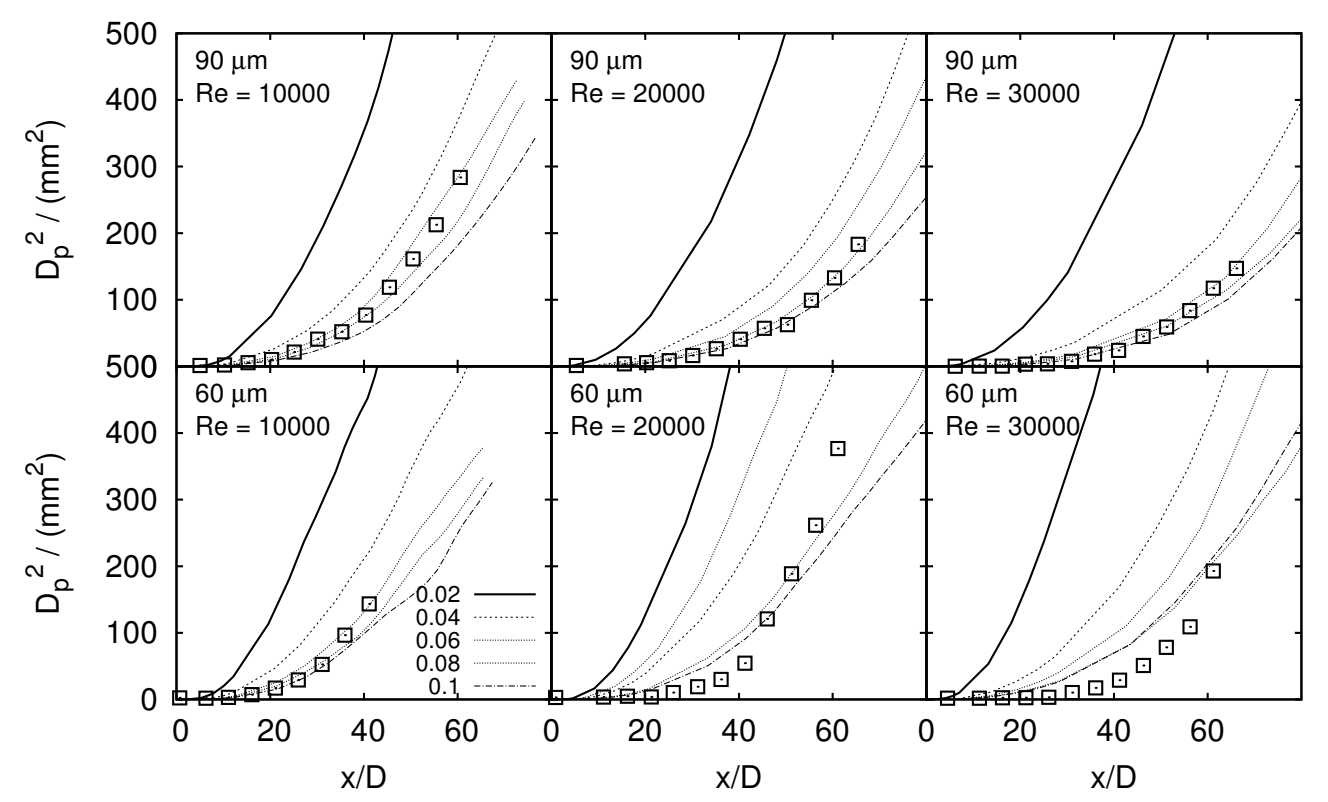

Figure 20: $\beta_{p}$ sensitivity on the Type-C dispersion of $60 \mu \mathrm{m}$ and $90 \mu \mathrm{m}$ particles in the $7 \mathrm{~mm}$ jet with $R e=10000,20000$ and 30000. Square symbols represent experimental measurements.

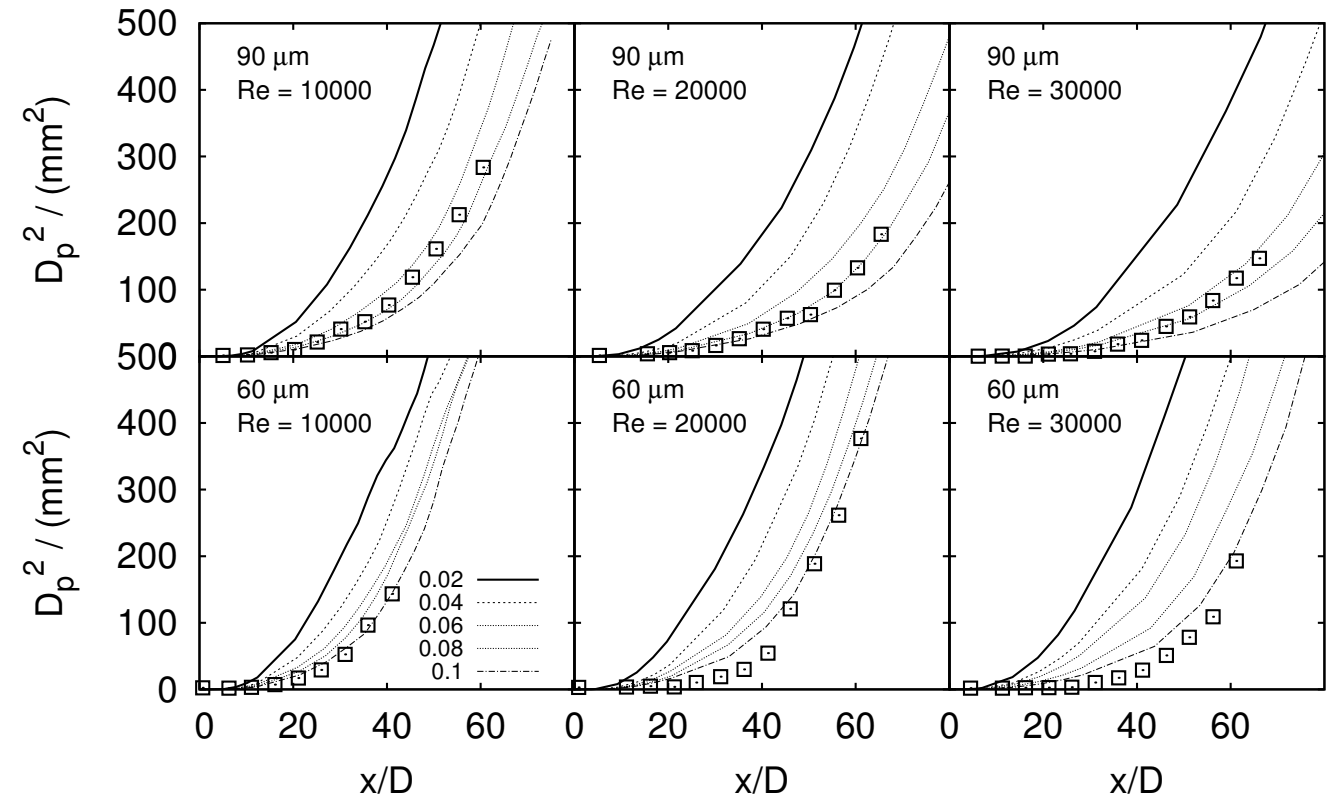

Figure 21: $\beta_{p}$ sensitivity on the Type-IC dispersion of $60 \mu \mathrm{m}$ and $90 \mu \mathrm{m}$ particles in the $7 \mathrm{~mm}$ jet with $R e=10000,20000$ and 30000. Square symbols represent experimental measurements. 


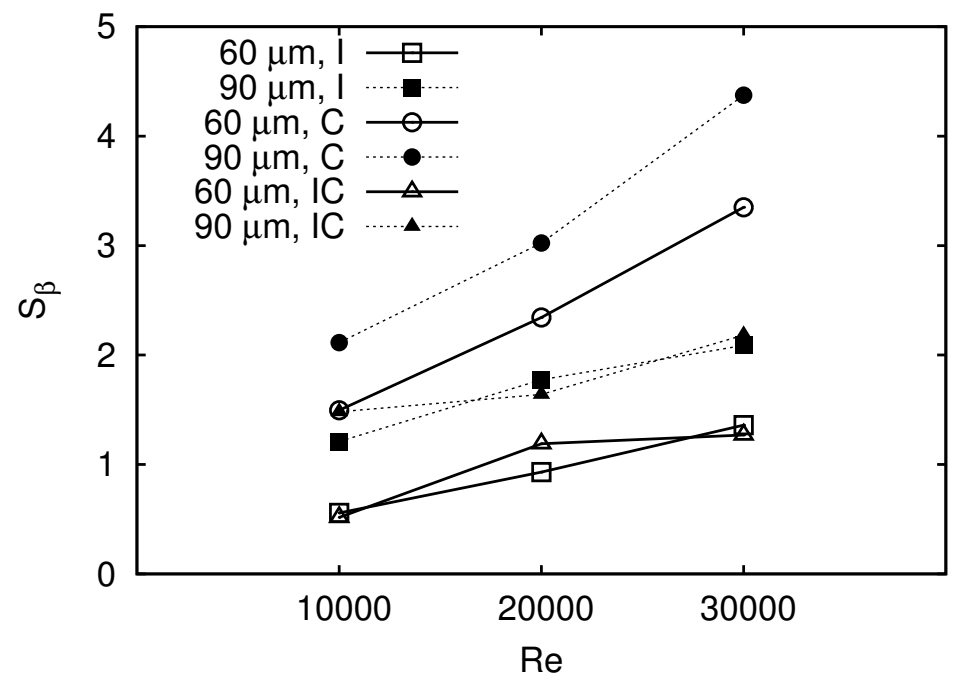

Figure 22: $\beta_{p}$ sensitivity to dispersion of $60 \mu \mathrm{m}$ and $90 \mu \mathrm{m}$ particles at $x / D=50$ in the $7 \mathrm{~mm}$ jet with $R e=10000,20000$ and 30000 .

where $D_{p}^{2}$ is particle dispersion evaluated at $x / D=50$, and $\beta_{p, 3}$ is the average value of $\beta_{p, 1}$ and $\beta_{p, 2}$. Large $S_{\beta}$ indicates high sensitivity of particle dispersion to $\beta_{p}$. Figure 22 shows $S_{\text {beta }}(0.02,0.1)$ as a function of $R e$ for the three different interaction models. The dispersion is more sensitive to $\beta_{p}$ for larger particles and at high $R e$ due to the increasing impact of the crossing-trajectory effect. This effect was observed in the previous study of multiphase homogeneous turbulence [34]. The Type-C interaction model is shown to be significantly more sensitive to $\beta_{p}$ than the two other models. This is because the Type-C model allows the particles to interact with multiple eddies simultaneously that leads to more trajectory crossings.

\section{Conclusions}

This study has been concerned with the development of ODT multiphase models coupling a dispersed Lagrangian particle phase to the fluid evolution using three particle-eddy interaction models (Type-I, -C, and $-\mathrm{IC}$ ), and the prediction of the transport of particles in turbulent round jet flows (and more general shear-driven turbulent flows). The challenge in this work is to properly account for particle-eddy interaction.

The ODT multiphase model uses a Lagrangian framework to solve the transport equations of a particle as it interacts with a succession of discrete turbulent eddies. The Type-I PEI model uses instantaneous particleeddy interactions and provides good predictions of particle dispersion, but does not allow the particles to interact with multiple eddies at the same time. The Type-C PEI model resolves the above drawback of the Type-I PEI model by using continuous PEIs for the finite evolution time. However, the Type-C model is not able to capture the tracer limit, and therefore only accurately predicts higher Stokes-number particles. The Type-IC PEI model combines the features of the Type-I and -C PEI models, and it is considered to be 
the most robust PEI model among the three.

The models compare favorably with experimental results for a range of characteristic particle response times and jet exit velocities. The particle dispersion in Lagrangian form is initially quadratic for short times-of-flight; the function becomes linear for long times-of-flight as the particle Stokes number becomes $O(1)$ and the particles behave more like tracer particles. The single model parameter $\beta_{p}$ is used to scale the eddy lifetime and fluid velocities felt by particles during the interactions. Particle interactions depend on the lesser of the eddy lifetime and the eddy-crossing time, and this makes dispersion results sensitive to $\beta_{p}$ for finite Stokes-number particles. The sensitivity was evaluated and is greater for larger particles and for the flows with greater overall acceleration (higher Reynolds number here) due to enhanced eddy crossing.

The ODT model has the benefit of resolving a full range of length and time scales with dynamically evolved turbulence properties. Hence, the ODT particle model is expected to provide a novel approach to modeling a wide range of dispersed particle flows, providing an alternative to methods that filter the fine scales. Extension of the models to reacting flows, and flows with particle modulation of turbulence under high particle loading conditions are under development.

\section{Acknowledgments}

The authors acknowledge helpful discussions with Alan Kerstein. This work was supported by the Defense Threat Reduction Agency under Award Number HDTRA-11-4503I. Sandia National Laboratories is a multiprogram laboratory managed and operated by Sandia Corporation, a wholly owned subsidiary of Lockheed Martin Corporation, for the U.S. Department of Energy's National Nuclear Security Administration under contract DE-AC04-94AL85000.

\section{References}

[1] Almeida, T. G., Jaberi, F. A., 2008. Large-eddy simulation of a dispersed particle-laden turbulent round jet. International Journal of Heat and Mass Transfer 51 (3), 683-695.

[2] Ashurst, W. T., Kerstein, A. R., 2005. One-dimensional turbulence: variable density formulation and application to mixing layers. Physics of Fluids 17-025107, 1-26.

[3] Batchelor, G., 1957. Diffusion in free turbulent shear flows. Journal of Fluid Mechanics 3 (01), 67-80.

[4] Berlemont, A., Desjonqueres, P., Gouesbet, G., 1990. Particle lagrangian simulation in turbulent flows. International Journal of Multiphase Flow 16 (1), 19-34.

[5] Chien, K., 1982. Predictions of channel and boundary-layer flows with a low-Reynolds-number turbulence model. AIAA Journal 20 (1), 33-38.

[6] Clift, R., Grace, J. R., Weber, M. E., 1978. Bubbles, drops and particles. Academic Press, New York.

[7] Csanady, G., 1963. Turbulent diffusion of heavy particles in the atmosphere. Journal of Atmospheric Science 20, $201-208$.

[8] Echekki, T., Kerstein, A. R., Dreeben, T. D., 2001. One-dimensional turbulence simulation of turbulent jet diffusion flames: model formulation and illustrative applications. Combustion and Flame 125, 1083-1105.

[9] Goshayeshi, B., Sutherland, J. C., 2015. A comparative study of themochemistry models for oxy-coal combustion simulation. Combustion and Flame 162 (10), 4016-4024.

[10] Goshayeshi, B., Sutherland, J. C., 2015. Prediction of oxy-coal flame stand-off using high-fidelity thermochemical models and the one-dimensioinal turbulence model. Proceedings of the Combustion Institute 35, $2829-2837$.

[11] Gosman, A., Loannides, E., 1983. Aspects of computer simulation of liquid-fueled combustors. Journal of Energy 7 (6), $482-490$.

[12] Hewson, J. C., Kerstein, A. R., 2001. Stochastic simulation of transport and chemical kinetics in turbulent CO/H $/ \mathrm{N}_{2}$ flames. Combustion Theory and Modelling 5, 669-697. 
[13] Hewson, J. C., Kerstein, A. R., 2002. Local extinction and reignition in nonpremixed turbulent $\mathrm{CO} / \mathrm{H}_{2} / \mathrm{N}_{2}$ jet flames. Combustion Science and Technology 174, 35-66.

[14] Iliopolous, I., Hanratty, T., 1999. Turbulent dispersion in a non-homogenous field. Journal of Fluid Mechanics 392, 45-71.

[15] Kennedy, I. M., Moody, M. H., 1998. Particle dispersion in a turbulent round jet. Experimental Thermal and Fluid Science $18,11-26$.

[16] Kerstein, A. R., 1999. One-dimensional turbulence: model formulation and application to homogeneous turbulence, shear flows, and buoyant stratified flows. Journal of Fluid Mechanics 392, 277-334.

[17] Kerstein, A. R., 2013. Hierarchical parcel-swapping representation of turbulent mixing. part 1. formulation and scaling properties. Journal of Statistical Physics 153 (1), 142-161.

[18] Kerstein, A. R., Ashurst, W. T., Wunsch, S., Nilsen, V., 2001. One-dimensional turbulence: vector formulation and application to free shear flows. Journal of Fluid Mechanics 447, 85-109.

[19] Lewis, P. A., Shedler, G. S., 1979. Simulation of nonhomogeneous poisson processes by thinning. Naval Res. Logistics Quart. 26, 403-413.

[20] Li, D., Fan, J., Luo, K., Cen, K., 2011. Direct numerical simulation of a particle-laden low reynolds number turbulent round jet. International Journal of Multiphase Flow 37 (6), 539-554.

[21] Lignell, D. O., Kerstein, A. R., Sun, G., Monson, E. I., 2012. Mesh adaption for efficient multiscale implementation of one-dimensional turbulence. Theoretical and Computational Fluid Dynamics.

[22] MacInnes, J., F.V., B., 1992. Stochastic particle dispersion modeling and the tracer-particle limit. Physics of Fluids A 4 (12), 2809-2824.

[23] Ormancey, A., Martinon, J., 1984. Prediction of particle dispersion in turbulent flows. PhysicoChemical Hydrodynamics $5,229-244$.

[24] Papoulis, A., Pillai, S. U., 2002. Probability, Random Variables, and Stochastic Processes, 4th Edition. McGraw-Hill, New York.

[25] Punati, N., 2012. An Eulerian one-dimensional turbulence model: application to turbulent and multiphase reacting flows. Ph.D. thesis, University of Utah.

[26] Ricks, A. J., Hewson, J. C., Kerstein, A. R., Gore, J. P., Tieszen, S. R., Ashurst, W. T., 2010. A spatially developing onedimensional turbulence (ODT) study of soot and enthalpy evolution in meter-scale buoyant turbulent flames. Combustion Science and Technology 182, 60-101.

[27] Schlichting, H., 1979. Boundary Layer Theory, 7th Edition. McGraw-Hill.

[28] Schmidt, J. R., 2004. Stochastic models for the prediction of individual particle trajectories in one dimensional turbulence flows. Ph.D. thesis, The University of Arizona.

[29] Schmidt, J. R., Wendt, J. O., Kerstein, A. R., 2006. Prediction of particle laden turbulent channel flow using onedimensional turbulence. In: IUTAM Symposium on Computational Approaches to Multiphase Flow. Springer, pp. 433441.

[30] Schmidt, J. R., Wendt, J. O., Kerstein, A. R., 2009. Non-equilibrium wall deposition of inertial particles in turbulent flow. Journal of Statistical Physics 137 (2), 233-257.

[31] Schmidt, J. R., Wendt, J. O. L., Kerstein, A. R., October 2004. Prediction of particle laden turbulent channel flow using one dimensional turbulence. In: Proceedins of the IUTAM Symposium on Computational Approaches to Disperse Multiphase Flow, Argonne, IL.

[32] Shirolkar, J., Coimbra, C., McQuay, M., 1996. Fundamental aspects of modeling turbulent particle dispersion in dilute flows. Progress in Energy and Combustion Science 22, 363-399.

[33] Shuen, J., Chen, L., Faeth, G., 1983. Evaluation of a stochastic model of particle dispersion in a turbulent round jet. AIChE Journal 29, 167-170.

[34] Sun, G., Lignell, D. O., Hewson, J. C., Gin, C. R., 2014. Particle dispersion in homogeneous turbulence using the onedimensional turbulence model. Physics of Fluids (1994-present) 26 (10), 103301.

[35] Taylor, G. I., 1922. Diffusion by continuous movements. Proc. London Math. Soc 20 (1), $196-212$.

[36] van Reeuwijk, M., Holzner, M., 2014. The turbulence boundary of a temporal jet. Journal of Fluid Mechanics $739,254-275$.

[37] Wunsch, S., Kerstein, A., 2001. A model for layer formation in stably stratified turbulence. Physics of Fluids 13 , 702-712.

[38] Yudine, M., 1959. Physical consideration on heavy particle diffusion. Advances in Geophysics 6, $185-191$.

[39] Yuu, S., Ueno, T., Umekage, T., 2001. Numerical simulation of the high reynolds number slit nozzle gas-particle jet using subgrid-scale coupling large eddy simulation. Chemical Engineering Science 56 (14), 4293-4307.

[40] Yuu, S., Yasukouchi, N., Hirosawa, Y., Jotaki, T., 1978. Particle turbulent diffusion in a dust laden round jet. AIChE Journal 24 (3), 509-519.

41] Zhou, Q., Leschziner, M., 1991. A time-correlated stochastic model for particle dispersion in anisotropic turbulence. In: 8th Symposium on Turbulent Shear Flows, Volume 1. Vol. 1. pp. 10-3. 